\title{
DISENTANGLING AGN AND STAR FORMATION IN SOFT X-RAYS
}

\author{
Stephanie M. LaMassa ${ }^{1,3}$, T. M. Heckman ${ }^{1}$, and A. PtaK ${ }^{2}$ \\ ${ }^{1}$ The Johns Hopkins University, Department of Physics \& Astronomy, Baltimore, MD 21218, USA \\ 2 NASA/Goddard Space Flight Center, Greenbelt, MD 20771, USA \\ Received 2012 June 27; accepted 2012 August 30; published 2012 September 28
}

\begin{abstract}
We have explored the interplay of star formation and active galactic nucleus (AGN) activity in soft X-rays (0.5-2 keV) in two samples of Seyfert 2 galaxies (Sy2s). Using a combination of low-resolution CCD spectra from Chandra and XMM-Newton, we modeled the soft emission of 34 Sy2s using power-law and thermal models. For the 11 sources with high signal-to-noise Chandra imaging of the diffuse host galaxy emission, we estimate the luminosity due to star formation by removing the AGN, fitting the residual emission. The AGN and star formation contributions to the soft X-ray luminosity (i.e., $L_{x, \mathrm{AGN}}$ and $L_{x, \mathrm{SF}}$ ) for the remaining 24 Sy2s were estimated from the power-law and thermal luminosities derived from spectral fitting. These luminosities were scaled based on a template derived from XSINGS analysis of normal star-forming galaxies. To account for errors in the luminosities derived from spectral fitting and the spread in the scaling factor, we estimated $L_{x}$, AGN and $L_{x}$, SF from Monte Carlo simulations. These simulated luminosities agree with $L_{x, \mathrm{AGN}}$ and $L_{x, \mathrm{SF}}$ derived from Chandra imaging analysis within a $3 \sigma$ confidence level. Using the infrared [Ne II] $12.8 \mu \mathrm{m}$ and [O IV] $26 \mu \mathrm{m}$ lines as a proxy of star formation and AGN activity, respectively, we independently disentangle the contributions of these two processes to the total soft X-ray emission. This decomposition generally agrees with $L_{x}$,SF and $L_{x}$, AGN at the $3 \sigma$ level. In the absence of resolvable nuclear emission, our decomposition method provides a reasonable estimate of emission due to star formation in galaxies hosting type 2 AGNs.
\end{abstract}

Key words: galaxies: Seyfert - galaxies: starburst - infrared: galaxies - X-rays: galaxies

Online-only material: color figures

\section{INTRODUCTION}

Supermassive black holes (SMBHs) and their parent galaxies co-evolve (Kormendy \& Richstone 1995; Magorrian et al. 1998; Ferrarese \& Merritt 2000; Gebhardt et al. 2000; Tremaine et al. 2002; Häring \& Rix 2004). In particular, observational and theoretical work has established a link between accreting SMBHs (active galactic nuclei or AGNs) and host galaxy star formation. Common mechanisms have been proposed for triggering star formation while fueling SMBH accretion, including galaxy mergers (e.g., Sanders et al. 1988; Hopkins et al. 2008) and secular processes involving gravitational instabilities induced by spiral arms or galactic-sized bars (e.g., Kormendy \& Kennicutt 2004; Cisternas et al. 2011; Schawinski et al. 2011).

Disentangling star formation from AGN activity becomes a necessary endeavor to investigate SMBH and host galaxy coevolution. This separation has been explored extensively and has included identifying optical and ultraviolet (UV) spectral signatures of starburst activity in AGNs (Cid Fernandes et al. 2001), performing principal component analysis on infrared spectra of AGNs (e.g., Buchanan et al. 2006), and analyzing optical and infrared (IR) diagnostics that parameterize the relative contribution of AGNs to star formation, such as ratios of infrared and optical emission lines that indicate ionization field hardness (Genzel et al. 1998; Kewley et al. 2001; Kauffmann et al. 2003; Treyer et al. 2010; LaMassa et al. 2010, 2012) and strength of the polycyclic aromatic hydrocarbon features (Genzel et al. 1998; O’Dowd et al. 2009; LaMassa et al. 2010, 2012). Here we extend the study of the interplay between AGN activity and star formation to the X-ray regime.

Quiescent galaxies emit thermal and non-thermal X-ray emission. Hot gas energized by stellar winds and supernova

\footnotetext{
S. M. LaMassa is now at Yale University.
}

explosions radiates in soft X-rays $(0.5-2 \mathrm{keV})$ whereas X-ray binaries $(\mathrm{XRBs})$ and supernova remnants dominate the nonthermal emission above $2 \mathrm{keV}$ (see Fabbiano 2006 for a review). Due to the relatively short life time of high mass X-ray binaries (HMXBs, $\tau<10^{7} \mathrm{yr}$ ) and the short delay between a starburst and the generation of soft X-rays from hot gas, hard and soft $\mathrm{X}$-ray emission can trace the instantaneous star formation rate (SFR). Indeed, X-ray emission from quiescent galaxies is well correlated with radio, infrared, optical, and ultraviolet SFR indicators (e.g., Ranalli et al. 2003; Rosa González et al. 2009; Pereira-Santaella et al. 2011). Various X-ray SFRs have thus been calibrated in the literature based on the correlation of X-ray emission with far-infrared and radio luminosities (Ranalli et al. 2003), X-ray emission from HMXBs and cumulative galactic $\mathrm{X}$-ray point sources with far-infrared luminosities (Persic et al. 2004; Persic \& Rephaeli 2007), and X-ray luminosity with infrared plus ultraviolet emission (Pereira-Santaella et al. 2011). However, low mass X-ray binaries, which trace stellar mass $\left(M_{\star}\right)$ rather than star formation, also contribute to the X-ray emission and become the primary source of X-ray radiation in galaxies with low SFR $/ M_{\star}$ (Colbert et al. 2004; Lehmer et al. 2010).

An active nucleus complicates using X-ray emission as an SFR indicator: accretion onto the SMBH dominates emission above $2 \mathrm{keV}$. Isolating the hard X-ray emission due to star formation then becomes prohibitively difficult when the nucleus is not resolved. However, soft X-ray emission can have comparable contributions from scattered/reflected AGN emission and thermal emission from gas associated with starburst activity when the AGN is obscured or has very low luminosity. Determining the relative contribution of AGNs and star formation to the soft X-ray emission can be achieved through highresolution spectroscopy which resolves narrow emission lines. Various diagnostics can be used to distinguish between collisionally and photoionized plasma, such as the width of the radiative 
recombination continua (Liedahl \& Paerels 1996) and ratios of forbidden, intercombination, and resonance lines in the O vir triplet (Porquet \& Dubau 2000). However, with current X-ray missions (i.e., Chandra and XMM-Newton) highresolution spectra are only obtainable through grating observations, necessitating long exposure times on X-ray-bright sources to achieve adequate signal-to-noise. How well can less timeintensive low-resolution CCD spectroscopy achieve the goal of disentangling starbursts from AGN activity in soft X-rays?

Local obscured AGNs, Seyfert 2 galaxies (Sy2s), provide an ideal laboratory for answering this question. As these sources are relatively nearby and the accretion disk is hidden behind an obscuring "torus," circumnuclear starbursts are visible. Hence, both AGN and host galaxy star formation can be modeled. Levenson et al. (2004, 2005) analyzed Chandra observations of two Sy2/starburst composites (NGC 5135 and NGC 7130) and demonstrated that a thermal component due to stellar processes was necessary to adequately model the soft X-ray emission. We expand this methodology to Sy2s in general to investigate the efficacy of modeling soft X-ray emission with a thermal component to describe host galaxy star formation.

Our analysis focuses on two samples of Sy2s: an optically selected [O III]5007 Å sample (LaMassa et al. 2009) and a midinfrared-selected $12 \mu \mathrm{m}$ sample (Spinoglio \& Malkan 1989). Using Chandra and XMM-Newton observations, we decomposed the soft X-ray emission into a star-forming and an AGN component. For Sy2s observed with Chandra that have significant host galaxy emission, we are able to resolve the point source associated with the AGN and can therefore remove it and analyze emission solely from the host galaxy, which we ascribe to star formation $\left(L_{x, \mathrm{SF}}\right)$. For the Sy2s observed with XMM-Newton and the remaining Chandra sources, we fit the X-ray spectra with a combination of a thermal and power-law model. To account for the presence of XRBs, we scale the soft thermal and power-law fluxes using star-forming galaxies from the XSINGS sample as a template (A. Ptak et al. 2012, in preparation), obtaining soft X-ray luminosity values associated with star formation $\left(L_{x, \mathrm{SF}}\right)$ and AGN activity $\left(L_{x, \mathrm{AGN}}\right)$. We compare these estimates of $L_{x, \mathrm{SF}}$ and $L_{x, \mathrm{AGN}}$ with infrared (IR) spectral signatures that accurately describe star formation ([Ne II]12.8 $\mu$ m luminosity, $L_{\text {[Ne II] }}$; Ho \& Keto 2007; LaMassa et al. 2012) and AGN activity

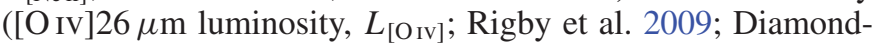
Stanic et al. 2009; Meléndez et al. 2008; LaMassa et al. 2010) to test the accuracy of this decomposition.

\section{SAMPLES AND DATA ANALYSIS}

The selection details for the [O III] and $12 \mu \mathrm{m}$ samples are presented in LaMassa et al. (2009) and Spinoglio \& Malkan (1989) and are therefore only briefly mentioned here. The [O III] sample was selected from Sloan Digital Sky Survey (SDSS) Data Release 4 (Adelman-McCarthy et al. 2006). These sources live in the Seyfert 2 locus of the BPT diagram (Baldwin et al. 1981) according to the Kewley et al. (2006) demarcation, and the sample is complete to a flux limit of $4 \times 10^{-14} \mathrm{erg} \mathrm{cm}^{-2} \mathrm{~s}^{-1}$, totaling 20 sources. Seventeen of these Sy2s were observed with $X M M-N e w t o n$. The $12 \mu \mathrm{m}$ sample is composed of Sy2s from the $I R A S$ point-source catalog, is complete to a flux density limit of $0.3 \mathrm{Jy}$ at $12 \mu \mathrm{m}$, and totals 31 galaxies. Twenty-eight of these sources have archival Chandra and/or XMM-Newton data.

In LaMassa et al. (2009, 2011) we discussed in detail the X-ray data reduction, using XAssist (Ptak \& Griffiths 2003), and broadband X-ray $(0.5-10 \mathrm{keV})$ spectral fitting of these sources with XSpec. Each galaxy was fit with an absorbed power law or when necessary, double absorbed power law, with the spectral indices initially tied together, simulating a partial covering geometry where a certain percentage of the transmitted $\mathrm{X}$-ray emission is absorbed and the remainder scattered into the line of sight; $\mathrm{Fe} \mathrm{K} \alpha$ emission, when present, was modeled with a Gaussian component. To accommodate the possible presence of starburst emission in soft X-rays $(0.5-2 \mathrm{keV})$, we included a thermal model (APEC) in these spectral fits which describes emission from a collisionally ionized plasma. Here we revisit more detailed modeling of the soft emission.

Since our aim is to study host galaxy star formation in tandem with AGN activity, we limit our analysis to the Sy2s where the addition of a thermal model component results in a detection of thermal emission, rather than an upper limit. As a result, NGC 291, CGCG 064-017, CGCG 218-007, and 2MASX J13463217+6423247 were dropped from the [O III] sample. We also exclude the three $12 \mu \mathrm{m}$ sources that were not detected in 2-10 keV X-rays: F08572+3915, NGC 5953, and NGC 7590. NGC 1068, part of the original $12 \mu \mathrm{m}$ sample, has soft X-ray emission dominated by AGN photoionization (e.g., Kinkhabwala et al. 2002) and has therefore been omitted from this analysis. In total, 38 Seyfert 2 galaxies remain: 14 from the [O III] sample and 24 from the $12 \mu \mathrm{m}$ sample.

As noted in LaMassa et al. (2011), several $12 \mu \mathrm{m}$ Sy2s had both Chandra and XMM-Newton archival observations. We tested if differences in the spectra for an individual source between the two observatories were due to aperture effects, since Chandra has a higher spatial resolution than XMM-Newton, or variability. Our aim in that analysis was to constrain the properties of the AGN, so we focused on the Chandra data to isolate the point source, using XMM-Newton information to help constrain the spectral fit for non-variable sources. In this analysis, we favor larger aperture extraction areas as they encompass extended X-ray emission due to host galaxy star formation. For the Sy2s in the $12 \mu \mathrm{m}$ sample with both XMMNewton and Chandra observations, the Chandra extraction area was adjusted to match the area from XMM-Newton or to encompass all visible extended X-ray emission. For the sources with just Chandra data, we increased the extraction area from the analysis presented in LaMassa et al. (2011) to ensure all extended X-ray emission is covered. In Tables 1 and 2, we list the aperture sizes used to extract spectra for the [O III] and $12 \mu \mathrm{m}$ Sy2s, respectively.

The spectral fitting results for the [O III] and $12 \mu \mathrm{m}$ samples are summarized in Tables 3 and 4. The broadband X-ray spectra $(0.5-10 \mathrm{keV})$ were fitted to most fully constrain the AGN contribution to the soft X-ray emission. Multiple spectra of the same source (from the three XMM-Newton detectors and/or multiple observations) were initially fit simultaneously with a multiplicative constant allowed to vary to account for differences in detector sensitivity. For variable sources, individual model parameters were fit independently. We imposed a lower column density $\left(N_{\mathrm{H}}\right)$ limit of that due to our Galaxy along the line of sight to the source. In some cases, the best-fit absorption pegged at this lower limit and $N_{\mathrm{H}}$ was subsequently frozen to this value. For the sources better described by a double power-law model, which as noted above represents a partial covering geometry, the photon indices were tied together, with the normalizations left free to vary and an independent absorption attenuating the second power-law component $\left(N_{\mathrm{H}, 2}\right)$; the Sy2s best accommodated with a single power law can be identified by the blank entry in the $N_{\mathrm{H}, 2}$ column of Tables 3 and 4 . The lower temperature 
Table 1

[O III] Sample: Aperture Extraction Areas ${ }^{\mathrm{a}}$

\begin{tabular}{|c|c|c|c|}
\hline Galaxy & $z$ & $\begin{array}{l}\text { Aperture Radius (") } \\
\text { PN/MOS1/MOS2 }\end{array}$ & $\begin{array}{c}\text { Aperture Radius }(\mathrm{kpc})^{\mathrm{b}} \\
\mathrm{PN} / \mathrm{MOS} 1 / \mathrm{MOS} 2\end{array}$ \\
\hline Mrk 0609 & 0.034 & $65 / 34 / 35$ & $44 / 23 / 24$ \\
\hline IC 0486 & 0.027 & $34 / 33 / 35$ & $19 / 18 / 19$ \\
\hline 2MASX J08035923+2345201 & 0.029 & $17 / 20 / 18$ & $10 / 12 / 11$ \\
\hline 2MASX J08244333+2959238 & 0.025 & $40 / 40 / 40$ & $21 / 21 / 21$ \\
\hline 2MASX J10181928+3722419 & 0.049 & $20 / 22 / 22$ & $20 / 22 / 22$ \\
\hline CGCG 242-028 & 0.026 & $19 / 22 / 13$ & $10 / 12 / 7$ \\
\hline SBS $1133+572$ & 0.050 & $20 / 16 / 17$ & $20 / 16 / 17$ \\
\hline Mrk 1457 & 0.049 & $19 / 13 / 13$ & $18 / 13 / 13$ \\
\hline 2MASX J11570483+5249036 & 0.036 & $35 / 35 / 35$ & $26 / 26 / 26$ \\
\hline 2 MASX J12183945+4706275 & 0.094 & $-/ 30 / 31$ & $53 / 55$ \\
\hline 2MASX J12384342+0927362 & 0.083 & $33 / 30 / 30$ & $52 / 47 / 47$ \\
\hline NGC 5695 & 0.014 & $31 / 22 / 17$ & $9 / 7 / 5$ \\
\hline
\end{tabular}

Notes.

a [O III] sources were observed with XMM-Newton only. PN, MOS1, and MOS2 refer to the three detectors on board XMM-Newton.

${ }^{\mathrm{b}}$ Conversion from arcseconds to kiloparsecs is based on the cosmology of $H_{0}=70 \mathrm{~km} \mathrm{~s}^{-1} \mathrm{Mpc}^{-1}, \Omega_{M}=0.27$, and $\Omega_{\Lambda}=0.73$.

${ }^{c}$ Only PN spectrum fit since MOS1 and MOS2 spectra suffered from low signal-to-noise.

${ }^{\mathrm{d}}$ Source fell on chip gap in PN detector so only spectra from MOS1 and MOS2 were fit.

limit $(k T)$ was set at $0.1 \mathrm{keV}$. Ten Sy2 galaxies were well fit by a second APEC component, significant at greater than the $3 \sigma$ level according to the $f$-test: 2MASX J08244333+2959238, NGC 424, NGC 1320, NGC 1386, NGC 4388, M-3-34-64, NGC 5194, Mrk 463, NGC 7582, and NGC 7674. This result indicates the presence of a two-temperature gas, similar to the results of X-ray observations of starburst galaxies (Dahlem et al. 1998; Strickland et al. 2004): one phase at $k T$ between $0.6-0.75 \mathrm{keV}$ and a lower temperature gas at $\sim 0.15 \mathrm{keV}$, with the exception of M-3-34-64 which has two hot gas components at $0.77_{-0.02}^{+0.02} \mathrm{keV}$ and $2.85_{-0.34}^{+0.34}$.

We list the observed soft fluxes in Tables 5 and 6: total 0.5-2 keV flux $\left(F_{0.5}-2 \mathrm{keV}\right)$, power-law component of soft X-ray flux $\left(F_{\text {pow }}\right)$, and APEC component of soft X-ray flux $\left(F_{\mathrm{APEC}}\right)$. In the subsequent analysis, we omit the Sy2s with a poorly constrained APEC flux, i.e., the sources where the error is an order of magnitude or more higher or lower than the measured value (2MASX J11110693+0228477 and CGCG 242-028), leaving us with 35 Sy2s.

\section{EFFECTS OF X-RAY BINARIES}

Before assigning "thermal" ( $F_{\text {APEC }}$ ) flux to starburst activity and power-law emission $\left(F_{\text {pow }}\right)$ to AGNs, we need to account for the effects of XRBs which contribute to the non-thermal emission. As noted above, HMXBs indicate instantaneous star formation in the host galaxy and this effect is incorporated into X-ray SFR calibrations. Since XRBs boost the nonthermal flux, $F_{\text {pow }}$ will overestimate the AGN emission while $F_{\text {APEC }}$ underrepresents host galaxy star formation. To assess the contribution of XRBs to the observable emission, we model just the extended emission when the AGN is resolvable. For the remaining sources, we apply a correction to $F_{\text {APEC }}$ and $F_{\text {pow }}$ using results of $\mathrm{X}$-ray analysis of star-forming galaxies from the XSINGS sample (A. Ptak et al. 2012, in preparation).

Spatially resolved emission from the narrow-line region (NLR) can also potentially affect the results, overpredicting the luminosity attributable to host galaxy star formation. For a handful of $12 \mu \mathrm{m}$ sources, the major radius $\left(R_{\text {maj }}\right)$ of the NLR was measured from Schmitt et al. (2003a), while we used the relations from Schmitt et al. (2003b) to estimate NLR $R_{\text {maj }}$ values for the remaining $12 \mu \mathrm{m}$ sources and the [O III] sample. For the [O III] Sy2s, the NLR radius ranges from 280 to $900 \mathrm{pc}$, with a median size of $\sim 0.5 \mathrm{kpc}$. Similarly, the $12 \mu \mathrm{m}$ selected Sy2s have NLR sizes ranging from 80 to $1320 \mathrm{pc}$, with a median $R_{\text {maj }}$ value of $\sim 0.39 \mathrm{kpc}$. For all [O III] and a majority of $12 \mu \mathrm{m}$ sources, the NLR is an order of magnitude or more smaller than the X-ray extraction radius. The NLR is thus likely not resolvable at these scales, so we only focus on quantifying the effects of XRBs on the observed emission. One notable exception is IC 5063, where the radius of the NLR $(\sim 1.3 \mathrm{kpc})$ comprises a significant fraction of the $\mathrm{X}$-ray extraction radius $(3 \mathrm{kpc})$. We will return to this Sy2 in Section 5. Another potential bias could be due to photoionized gas from the NLR being indistinguishable from collisionally ionized gas at low CCD resolution. However, as we show in Section 5, such an effect does not systematically affect our results.

\subsection{Chandra Imaging}

Due to Chandra's arcsecond resolution and the close proximity of the $12 \mu \mathrm{m} \mathrm{Sy} 2 \mathrm{~s}$, the central point source (i.e., AGN) is resolvable for a subset of our sample. To accurately constrain the AGN position, we ran wavedetect on 2-10 keV Chandra images of the $12 \mu \mathrm{m}$ sample. Spectra were then extracted from the remaining emission, omitting the AGN identified by wavedetect. Similar to above, the broadband X-ray emission was fitted with (an) absorbed power law(s) and an APEC component. We only include those sources where the spectra from the residual emission have a high enough signal-to-noise to be fit with the $\chi^{2}$ statistic (minimum grouping of 10 counts per bin), therefore dropping the sources where the X-ray emission is concentrated in the nucleus: NGC 424, TOLOLO 1238-364, F05189-2524, and NGC 5347. The summary of the spectral fitting for these 11 sources is listed in Table 7, and the associated total soft X-ray flux from the unresolved emission (i.e., host galaxy emission after AGN removal) is listed in the second column of Table 6. To first order, the total soft X-ray flux from the unresolved emission can be considered an estimate of host galaxy star formation, $L_{x, \mathrm{SF}} . L_{x, \mathrm{AGN}}$ is then estimated by taking the difference between 
Table 2

$12 \mu \mathrm{m}$ Sample: Aperture Extraction Areas ${ }^{\mathrm{a}}$

\begin{tabular}{|c|c|c|c|c|c|}
\hline Galaxy & $z$ & Observatory & $\begin{array}{c}\text { ObsID } \\
\mathrm{PN} / \mathrm{MOS} 1 / \mathrm{MOS} 2\end{array}$ & $\begin{array}{l}\text { Aperture Radius (") } \\
\text { PN/MOS1/MOS2 }\end{array}$ & Aperture Radius $(\mathrm{kpc})^{\mathrm{b}}$ \\
\hline \multirow[t]{2}{*}{ NGC 0424} & 0.012 & XMM-Newton & 00029242301 & $34 / 33 / 33$ & $8 / 7 / 7$ \\
\hline & & Chandra & 03146 & 15 & 3 \\
\hline NGC 1144 & 0.028 & XMM-Newton & 0312190401 & $36 / 33 / 33$ & $20 / 18 / 18$ \\
\hline NGC 1320 & 0.009 & XMM-Newton & 0405240201 & $37 / 35 / 35$ & 6/6/6 \\
\hline \multirow[t]{2}{*}{ NGC 1386} & 0.003 & XMM-Newton & 0140950201 & $45 / 40 / 40$ & $2 / 2 / 2$ \\
\hline & & Chandra & 04076 & 19 & 1 \\
\hline NGC 1667 & 0.015 & XMM-Newton & 0200660401 & $32 / 22 / 34$ & $10 / 7 / 10$ \\
\hline \multirow[t]{3}{*}{ F05189-2524 } & 0.043 & XMM-Newton & 0085640101 & $41 / 31 / 33$ & $35 / 26 / 28$ \\
\hline & & Chandra & 02034 & 40 & 34 \\
\hline & & Chandra & 03432 & 40 & 34 \\
\hline \multirow[t]{2}{*}{ NGC 3982} & 0.004 & XMM-Newton & 0204651201 & $34 / 37 / 44$ & $3 / 3 / 4$ \\
\hline & & Chandra & 04845 & 34 & 3 \\
\hline \multirow[t]{3}{*}{ NGC 4388} & 0.008 & XMM-Newton & 0110930701 & $36 / 35 / 43$ & $7 / 7 / 8$ \\
\hline & & XMM-Newton & 0110930301 & $37 / 32 / 33$ & $7 / 6 / 6$ \\
\hline & & Chandra & 01619 & 36 & 7 \\
\hline \multirow[t]{2}{*}{ NGC 4501} & 0.008 & XMM-Newton & 0112550801 & $18 / 22 / 33$ & $3 / 4 / 6$ \\
\hline & & Chandra & 02922 & 20 & 4 \\
\hline TOLOLO 1238-364 & 0.011 & Chandra & 04844 & 20 & 5 \\
\hline \multirow[t]{2}{*}{ NGC 4968} & 0.010 & XMM-Newton & 0002940101 & $17 / 18 / 14$ & $4 / 4 / 3$ \\
\hline & & XMM-Newton & 0200660201 & $21 / 18 / 18$ & $5 / 4 / 4$ \\
\hline M-3-34-64 & 0.017 & XMM-Newton & 0206580101 & $59 / 34 / 38$ & $21 / 12 / 14$ \\
\hline NGC 5135 & 0.014 & Chandra & 02187 & 20 & 6 \\
\hline \multirow[t]{6}{*}{ NGC $5194^{c}$} & 0.002 & XMM-Newton & 0112840201 & $65 / 42 / 49$ & $3 / 2 / 2$ \\
\hline & & XMM-Newton & 0303420101 & $-/ 56 / 49$ & $-/ 2 / 2$ \\
\hline & & XMM-Newton & 0303420201 & $-/ 61 / 46$ & $-/ 3 / 2$ \\
\hline & & Chandra & 00354 & 40 & 2 \\
\hline & & Chandra & 01622 & 40 & 2 \\
\hline & & Chandra & 03932 & 40 & 2 \\
\hline NGC 5347 & 0.008 & Chandra & 04867 & 6 & 1 \\
\hline \multirow[t]{2}{*}{ Mrk 463} & 0.050 & XMM-Newton & 0094401201 & $49 / 45 / 45$ & $49 / 45 / 45$ \\
\hline & & Chandra & 04913 & 40 & 40 \\
\hline \multirow[t]{8}{*}{ NGC $5506^{\mathrm{d}}$} & 0.006 & XMM-Newton & 0013140101 & $60 /-/-$ & $9 /-/-$ \\
\hline & & XMM-Newton & 0013140201 & $54 /-/-$ & $8 /-/-$ \\
\hline & & XMM-Newton & 0201830201 & $56 /-/ 53$ & $8 /-/ 8$ \\
\hline & & XMM-Newton & 0201830301 & $52 /-/ 64$ & $8 /-/ 9$ \\
\hline & & XMM-Newton & 0201830401 & $55 /-/ 55$ & $8 /-/ 8$ \\
\hline & & XMM-Newton & 0201830501 & $68 /-/ 98$ & $10 /-/ 14$ \\
\hline & & XMM-Newton & 0554170201 & $64 /-/-$ & 9/-/- \\
\hline & & XMM-Newton & 0554170101 & $66 /-/-$ & $10 /-/-$ \\
\hline \multirow[t]{4}{*}{ Arp 220} & 0.018 & XMM-Newton & 0101640801 & $20 / 21 / 25$ & $8 / 8 / 9$ \\
\hline & & XMM-Newton & 0101640901 & $28 / 25 / 23$ & $11 / 9 / 9$ \\
\hline & & XMM-Newton & 0205510201 & $34 / 28 / 22$ & $13 / 11 / 8$ \\
\hline & & Chandra & 00869 & 40 & 15 \\
\hline NGC 6890 & 0.008 & XMM-Newton & 0301151001 & $17 / 13 / 15$ & $3 / 2 / 2$ \\
\hline IC 5063 & 0.011 & Chandra & 07878 & 12 & 3 \\
\hline NGC 7130 & 0.016 & Chandra & 02188 & 17 & 5 \\
\hline \multirow[t]{3}{*}{ NGC 7172} & 0.009 & XMM-Newton & 0147920601 & $52 / 41 / 34$ & $8 / 7 / 5$ \\
\hline & & XMM-Newton & 0202860101 & $61 / 59 / 60$ & $10 / 9 / 10$ \\
\hline & & XMM-Newton & 0414580101 & $53 / 59 / 55$ & $8 / 9 / 9$ \\
\hline \multirow[t]{4}{*}{ NGC 7582} & 0.005 & XMM-Newton & 0112310201 & $44 / 41 / 41$ & $4 / 4 / 4$ \\
\hline & & XMM-Newton & 0204610101 & $60 / 43 / 47$ & $6 / 4 / 4$ \\
\hline & & Chandra & 00436 & 43 & 4 \\
\hline & & Chandra & 02319 & 43 & 4 \\
\hline NGC 7674 & 0.029 & XMM-Newton & 0200660101 & $19 / 18 / 16$ & $11 / 10 / 9$ \\
\hline
\end{tabular}

Notes.

a For XMM-Newton observations, the extraction areas for the PN, MOS1, and MOS2 detectors are listed separately.

${ }^{\mathrm{b}}$ Conversion from arcseconds to kiloparsecs is based on the cosmology of $H_{0}=70 \mathrm{~km} \mathrm{~s}^{-1} \mathrm{Mpc}^{-1}, \Omega_{M}=0.27$, and $\Omega_{\Lambda}=0.73$.

c Source fell on chip gap on PN detector for ObsIDs 0303420101 and 0303420201 ; these spectra were not fit.

${ }^{\mathrm{d}}$ Dashes indicate that the spectra suffered from pile-up in that particular detector and were therefore not fit. See LaMassa et al. (2011) for details. 
Table 3

[O III] Sample: APEC Model Parameters (Solar Abundance)

\begin{tabular}{|c|c|c|c|c|c|c|c|}
\hline Galaxy & $\begin{array}{c}N_{\mathrm{H}, 1} \\
\left(10^{22} \mathrm{~cm}^{-2}\right)\end{array}$ & $\begin{array}{c}k T_{1} \\
(\mathrm{keV})\end{array}$ & $\begin{array}{c}k T_{2} \\
(\mathrm{keV})\end{array}$ & $\Gamma$ & $\begin{array}{c}N_{\mathrm{H}, 2} \\
\left(10^{22} \mathrm{~cm}^{-2}\right)\end{array}$ & $\begin{array}{c}\chi^{2} 2 \text { APECs } \\
\text { DOF }\end{array}$ & $\begin{array}{c}\chi^{2} 1 \text { APEC } \\
\text { DOF }\end{array}$ \\
\hline Mrk 0609a & 0.04 & $0.27_{-0.04}^{+0.05}$ & $\ldots$ & $1.77_{-0.04}^{+0.05}$ & $\ldots$ & $\ldots$ & $160(203)$ \\
\hline IC 0486 & $<0.06$ & $<0.16$ & $\cdots$ & $1.23_{-0.07}^{+0.08}$ & $1.00_{-0.09}^{+0.10}$ & $\cdots$ & $636(629)$ \\
\hline 2MASX J08035923+2345201 & $0.61_{-0.14}^{+0.10}$ & $<0.12$ & $\cdots$ & $2.84_{-1.18}^{+1.05}$ & $46.7_{-24.1}^{+22.6}$ & $\cdots$ & $120(95)$ \\
\hline 2MASX J08244333+2959238 & 0.03 & $0.68_{-0.06}^{+0.06}$ & $<0.12$ & $1.54_{-0.34}^{+0.36}$ & $15.5_{-2.4}^{+2.8}$ & $229(186)$ & $263(188)$ \\
\hline $2 M A S X ~ J 10181928+3722419^{a}$ & 0.01 & $0.18_{-0.05}^{+0.04}$ & $\ldots$ & $2.63_{-0.69}^{+0.64}$ & $\ldots$ & $\cdots$ & $52.1(61)$ \\
\hline 2MASX J11110693+0228477 c & $<0.62$ & $0.23_{-0.09}^{+0.15}$ & $\ldots$ & $2.04_{-0.88}^{+1.88}$ & $\ldots$ & $\ldots$ & $37.5(36)$ \\
\hline CGCG $242-028^{b}$ & $0.69_{-0.23}^{+0.17}$ & $<0.15$ & $\cdots$ & $0.31_{-0.49}^{+0.46}$ & $\cdots$ & $\cdots$ & $87.0(90)$ \\
\hline SBS $1133+572$ & $<0.10$ & $0.824_{-0.21}^{+0.23}$ & $\ldots$ & $3.08_{-0.38}^{+0.61}$ & $57.6_{-30.2}^{+45.4}$ & $\ldots$ & $38.1(48)$ \\
\hline Mrk 1457 & $0.66_{-0.12}^{+0.12}$ & $0.14_{-0.03}^{+0.03}$ & $\ldots$ & $1.29_{-1.14}^{+1.37}$ & $27.5_{-9.3}^{+15.8}$ & $\ldots$ & $35.2(35)$ \\
\hline 2MASX J11570483+5249036 & $<0.1$ & $0.17_{-0.05}^{+0.03}$ & $\cdots$ & $2.85_{-0.34}^{+0.23}$ & $83.9_{-27.9}^{+47.1}$ & $\cdots$ & $123(76)$ \\
\hline 2 MASX J12183945+4706275 & 0.02 & $<0.24$ & $\cdots$ & $1.95_{-0.86}^{+0.70}$ & $87.2_{-34.1}^{+66.8}$ & $\cdots$ & $15(19)$ \\
\hline 2MASX J12384342+0927362 & $<0.07$ & $<0.23$ & $\ldots$ & $2.17_{-0.22}^{+0.31}$ & $29.3_{-2.6}^{+3.1}$ & $\cdots$ & 195 (164) \\
\hline NGC 5695 & $<0.59$ & $0.24_{-0.12}^{+0.73}$ & $\ldots$ & $2.55_{-0.52}^{+0.68}$ & $\ldots$ & $\ldots$ & 75.7 (62) \\
\hline
\end{tabular}

Notes.

${ }^{a}$ Best-fit absorption same as Galactic absorption. This parameter was then frozen to the Galactic value.

${ }^{\mathrm{b}}$ Used c-stat.

c PN only, MOS1 and MOS2 had low signal-to-noise spectra.

$L_{x, \mathrm{SF}}$ and $L_{0.5-2 \mathrm{keV}}$ for these Sy $2 \mathrm{~s}$, with the associated error derived by scaling the error of total soft X-ray luminosity by the ratio of the AGN luminosity to the total luminosity.

\subsection{Star-forming Galaxy Template}

We have used the results of XSINGS to estimate the contribution of XRBs to the soft X-ray emission in host galaxies. The XSINGS project entails the analysis of 96 Chandra observations of 56 SINGS galaxies (Kennicutt et al. 2003). SINGS is designed to cover a wide range of galaxy properties such as morphological type, SFR, stellar mass, and metallicity, and the main aim of XSINGS is to study the relationships between X-ray emission (XRBs, nuclear activity, and hot interstellar medium) and these properties. In A. Ptak et al. (2012, in preparation) the spatially averaged X-ray emission of XSINGS galaxies is discussed and compared to the summed XRB luminosities for galaxies with significant detections (XRB catalogs for all XSINGS galaxies will be presented in Jenkins et al. 2013, in preparation). XRBs emit as a power law, but provide a measure of the instantaneous SFR along with thermal emission in soft X-rays. We therefore have

$$
\begin{gathered}
L_{x, \mathrm{SF}}=L_{\mathrm{APEC}}+L_{\mathrm{XRB}} \\
L_{x, \mathrm{AGN}}=L_{\mathrm{pow}}-L_{\mathrm{XRB}} .
\end{gathered}
$$

We further assume that a certain fraction of X-ray emission from XRBs contributes to host galaxy star formation:

$$
L_{\mathrm{XRB}}=R \times L_{x, \mathrm{SF}} .
$$

Using the 22 pure star-forming galaxies in XSINGS (i.e., non Sy2s and non LINERs), we have calculated the mean ratio of $\mathrm{X}$-ray emission from resolved non-nuclear point sources (i.e., XRBs) to total X-ray emission in the $0.5-2 \mathrm{keV}$ band $(R)$, deriving $R=0.51 \pm 0.26$. Solving the above equations, we obtain

$$
L_{x, \mathrm{SF}}=\frac{L_{\mathrm{APEC}}}{1-R}
$$

$$
L_{x, \mathrm{AGN}}=L_{\mathrm{pow}}-R \frac{L_{\mathrm{APEC}}}{1-R} .
$$

To account for uncertainties in the luminosities derived from spectral fitting (i.e., $L_{\mathrm{APEC}}$ and $L_{\mathrm{pow}}$ ) and the wide spread in $R$ values, we have performed Monte Carlo simulations to derive $L_{x, \mathrm{SF}}$ and $L_{x, \mathrm{AGN}}$. We have drawn 1000 random values for $L_{\mathrm{APEC}}, L_{\mathrm{pow}}$, and $R$ from a Gaussian distribution centered on the best-fit values of $L_{\mathrm{APEC}}$ and $L_{\mathrm{pow}}$ (and the mean value of $R$ ), with $\sigma$ corresponding to the errors derived from spectral fitting (and standard deviation on $R$ ). Using these simulated parameters and Equations (4) and (5), we calculated 1000 simulated values for $L_{x, \mathrm{SF}}$ and $L_{x, \mathrm{AGN}}$. The mode of these distributions is then taken as $L_{x, \mathrm{SF}}$ and $L_{x, \mathrm{AGN}}$, with the $68 \%$ confidence interval as the associated errors.

For the subset of Sy2s with high signal-to-noise Chandra spectra of the soft unresolved emission, we compare the results of $L_{x, \mathrm{SF}}\left(L_{x, \mathrm{AGN}}\right)$ derived via Chandra imaging analysis and the simulation approach described above. Here, $L_{x}$,SF,Chandra ( $\left.L_{x, \mathrm{AGN}, \mathrm{Chandra}}\right)$ represents the soft X-ray luminosity due to star formation (AGN) based on spectral fitting of just the unresolved emission, while $L_{x, \mathrm{SF}, \mathrm{XSINGS}}\left(L_{x, \mathrm{AGN}, \mathrm{XSINGS}}\right)$ is the estimate of the star formation (AGN) component of the soft X-ray flux calculated via simulations.

To account for errors in both parameters, we use a Bayesian approach to linear regression (Kelly 2007). Both here and in Section 5, the high and low error bars on the X-ray flux were averaged to provide symmetric errors for the linear regression routine. We plot $L_{x, \mathrm{SF}, \mathrm{XSINGS}}$ as a function of $L_{x, \mathrm{SF} \text {,Chandra }}$ and $L_{x, \mathrm{AGN}, \mathrm{XSINGS}}$ against $L_{x, \mathrm{AGN} \text {,Chandra }}$ in Figure 1 . The gray shaded regions enclosed by the dashed lines delineate the $3 \sigma$ confidence level on the regression line, based on the median of the posterior distribution of the slope, intercept, and mean distribution of the independent variable, with the dot-dashed line denoting the line of equality. Though the $L_{x, \mathrm{SF}, \mathrm{XSINGS}}$ values are somewhat systematically higher than $L_{x, \mathrm{SF} \text {,Chandra }}$, they do agree within the $3 \sigma$ contours. The agreement among these independent methods suggests that using XSINGS galaxies as a template to correct the $L_{\mathrm{APEC}}$ and $L_{\text {pow }}$ values for the 
Table 4

APEC Model Parameters (Solar Abundance ${ }^{\mathrm{a}}$ )

\begin{tabular}{|c|c|c|c|c|c|c|c|}
\hline Galaxy & $\begin{array}{c}N_{\mathrm{H}, 1} \\
\left(10^{22} \mathrm{~cm}^{-2}\right)\end{array}$ & $\begin{array}{c}k T_{1} \\
(\mathrm{keV})\end{array}$ & $\begin{array}{c}k T_{2} \\
(\mathrm{keV})\end{array}$ & $\Gamma$ & $\begin{array}{c}N_{\mathrm{H}, 2} \\
\left(10^{22} \mathrm{~cm}^{-2}\right)\end{array}$ & $\begin{array}{c}\chi^{2} 2 \text { APECs } \\
\text { DOF }\end{array}$ & $\begin{array}{c}\chi^{2} 1 \text { APEC } \\
\text { DOF }\end{array}$ \\
\hline NGC 0424 & $0.04_{-0.03}^{+0.03}$ & $0.69_{-0.05}^{+0.08}$ & $<0.13$ & $2.21_{-0.28}^{+0.21}$ & $32.6_{-6.0}^{+8.5}$ & $246(178)$ & $267(180)$ \\
\hline NGC $1144^{\mathrm{a}}$ & 0.06 & $0.37_{-0.06}^{+0.29}$ & $\ldots$ & $1.91_{-0.24}^{+0.37}$ & $47.0_{-3.2}^{+3.5}$ & $\ldots$ & $175(149)$ \\
\hline NGC 1320 & $<0.08$ & $0.15_{-0.03}^{+0.03}$ & $0.73_{-0.02}^{+0.09}$ & $2.97_{-0.21}^{+0.27}$ & $48.0_{-15.5}^{+35.6}$ & $233(188)$ & $279(190)$ \\
\hline NGC 1386 & $0.04_{-0.01}^{+0.02}$ & $0.67_{-0.03}^{+0.03}$ & $0.13_{-0.01}^{+0.02}$ & $2.72_{-0.14}^{+0.12}$ & $31.4_{-11.5}^{+22.9}$ & $398(332)$ & $435(334)$ \\
\hline NGC $1667^{b}$ & 0.05 & $0.33_{-0.04}^{+0.07}$ & $\ldots$ & $2.18_{-0.37}^{+0.34}$ & $\ldots$ & $\cdots$ & $49.8(38)$ \\
\hline $\mathrm{F} 05189-2524^{\mathrm{b}}$ & 0.02 & 0.1 & $\ldots$ & $2.05_{-0.14}^{+0.14}$ & $6.77_{-0.42}^{+0.44}$ & $\ldots$ & $501(374)$ \\
\hline NGC $3982^{b}$ & 0.01 & $0.29_{-0.03}^{+0.03}$ & $\cdots$ & $2.39_{-0.15}^{+0.18}$ & $40.3_{-16.3}^{+25.5}$ & $\cdots$ & $174(160)$ \\
\hline NGC $4388(X M M)^{\mathrm{b}}$ & 0.03 & $0.60_{-0.03}^{+0.04}$ & $0.15_{-0.03}^{+0.02}$ & $1.25_{-0.12}^{+0.12}$ & $24.3_{-1.0}^{+1.1}$ & $510(495)$ & $580(498)$ \\
\hline NGC $4388(\text { Chandra })^{\mathrm{b}}$ & 0.03 & $0.60_{-0.04}^{+0.04}$ & $0.15_{-0.02}^{+0.04}$ & $0.38_{-0.30}^{+0.29}$ & $25.6_{-2.9}^{+3.1}$ & $210(166)$ & $269(168)$ \\
\hline NGC $4501^{b}$ & 0.03 & $0.36_{-0.03}^{+0.05}$ & $\ldots$ & $1.52_{-0.29}^{+0.30}$ & $\cdots$ & $\cdots$ & $94.2(102)$ \\
\hline TOLOLO $1238-364^{\mathrm{c}}$ & $<0.19$ & $0.32_{-0.06}^{+0.44}$ & $\ldots$ & $2.45_{-0.36}^{+0.33}$ & $\ldots$ & $\ldots$ & $91.6(105)$ \\
\hline NGC $4968^{b, c}$ & 0.08 & $0.68_{-0.12}^{+0.12}$ & $\cdots$ & $1.72_{-0.21}^{+0.18}$ & $\cdots$ & $\cdots$ & $325(268)$ \\
\hline$M-3-34-64^{b}$ & 0.05 & $2.85_{-0.38}^{+0.34}$ & $0.77_{-0.02}^{+0.02}$ & $3.24_{-0.23}^{+0.19}$ & $53.6_{-3.6}^{+2.9}$ & $780(492)$ & $848(493)$ \\
\hline NGC $5135^{b}$ & 0.05 & $0.73_{-0.03}^{+0.03}$ & $\ldots$ & $2.34_{-0.08}^{+0.07}$ & $\ldots$ & $\ldots$ & $166(114)$ \\
\hline NGC 5194 & $0.04_{-0.01}^{+0.01}$ & $0.18_{-0.01}^{+0.01}$ & $0.62_{-0.01}^{+0.01}$ & $3.18_{-0.14}^{+0.14}$ & $10.2_{-0.75}^{+0.80}$ & $1560(1291)$ & $2021(1293)$ \\
\hline NGC $5347^{\mathrm{b}, \mathrm{c}}$ & 0.02 & $<0.21$ & $\ldots$ & $1.53_{-0.29}^{+0.30}$ & $32.6_{-19.6}^{+24.1}$ & $\ldots$ & $69.9(82)$ \\
\hline $\operatorname{Mrk} 463^{\mathrm{b}}$ & 0.02 & $0.74_{-0.02}^{+0.04}$ & $0.20_{-0.04}^{+0.06}$ & $1.76_{-0.16}^{+0.13}$ & $28.6_{-3.4}^{+4.5}$ & 365 (316) & 392 (318) \\
\hline NGC $5506^{\mathrm{d}}$ & $0.11_{-0.01}^{+0.01}$ & $0.74_{-0.05}^{+0.05}$ & $\ldots$ & $1.71_{-0.01}^{+0.02}$ & $2.69_{-0.03}^{+0.02}$ & $\cdots$ & $2646(2380)$ \\
\hline NGC $5506^{\mathrm{e}}$ & $"$ & $0.94_{-0.24}^{+0.39}$ & $"$ & " & $"$ & $"$ & $"$ \\
\hline NGC $5506^{\mathrm{f}}$ & $0.17_{-0.01}^{+0.01}$ & $\ldots$ & $\ldots$ & $1.83_{-0.02}^{+0.02}$ & $2.80_{-0.02}^{+0.01}$ & $\ldots$ & 3982 (3137) \\
\hline NGC $5506^{\mathrm{g}}$ & $"$ & " & $"$ & $1.76_{-0.00}^{+0.01}$ & " & $"$ & " \\
\hline NGC $5506^{\mathrm{h}}$ & $0.12_{-0.01}^{+0.01}$ & $0.83_{-0.03}^{+0.03}$ & $"$ & " & " & $"$ & " \\
\hline NGC $5506^{\mathrm{i}}$ & $"$ & $0.96_{-0.05}^{+0.05}$ & $"$ & $"$ & $"$ & $"$ & $"$ \\
\hline Arp $220^{a, b}$ & 0.04 & $0.79_{-0.04}^{+0.04}$ & $\ldots$ & $0.95_{-0.24}^{+0.26}$ & $\ldots$ & $\ldots$ & $309(302)$ \\
\hline NGC $6890^{c}$ & $<0.22$ & $0.78_{-0.19}^{+0.24}$ & $\ldots$ & $3.28_{-0.74}^{+0.88}$ & $27.4_{-11.3}^{+18.4}$ & $\cdots$ & $164(148)$ \\
\hline IC $5063^{b, j}$ & 0.06 & $0.60_{-0.11}^{+0.10}$ & $\ldots$ & $1.85_{-0.21}^{+0.16}$ & $21.0_{-1.2}^{+1.1}$ & $\cdots$ & $198(141)$ \\
\hline NGC $7130^{c}$ & $0.06_{-0.02}^{+0.03}$ & $0.63_{-0.03}^{+0.03}$ & $\ldots$ & $2.43_{-0.18}^{+0.25}$ & $75.4_{-38.1}^{+55.6}$ & $\ldots$ & $334(240)$ \\
\hline NGC $7172^{b}$ & 0.02 & $0.28_{-0.04}^{+0.05}$ & $\ldots$ & $1.56_{-0.03}^{+0.02}$ & $7.36_{-0.10}^{+0.10}$ & $\ldots$ & 2074 (1746) \\
\hline NGC $7172^{b}$ & $"$ & $0.26_{-0.02}^{+0.02}$ & $\ldots$ & $1.54_{-0.03}^{+0.02}$ & $8.13_{-0.11}^{+0.11}$ & $"$ & " \\
\hline NGC $7582(\text { Chandra })^{\mathrm{b}}$ & 0.01 & $0.72_{-0.05}^{+0.05}$ & $\ldots$ & $1.94_{-0.10}^{+0.11}$ & $24.6_{-1.6}^{+1.8}$ & $\ldots$ & $355(301)$ \\
\hline NGC $7582(X M M)^{\mathrm{b}}$ & 0.01 & $0.18_{-0.04}^{+0.02}$ & $0.72_{-0.01}^{+0.01}$ & $1.75_{-0.03}^{+0.05}$ & $27.0_{-1.5}^{+1.5}$ & $1438(886)$ & $1586(888)$ \\
\hline NGC $7674^{\mathrm{b}, \mathrm{c}}$ & 0.04 & $<0.11$ & $0.67_{-0.05}^{+0.07}$ & $0.62_{-0.43}^{+0.47}$ & $89.0_{-40.2}^{+69.0}$ & $66.6(70)$ & $113(72)$ \\
\hline
\end{tabular}

Notes.

a All abundances frozen to solar except for Arp 220 which has an abundance of $0.17_{-0.05}^{+0.12}$.

b Best-fit absorption same as Galactic absorption. This parameter was then frozen to the Galactic value.

c Used c-stat.

d ObsIDs 0201830201, 0201830301, and 0201830401. APEC and first power-law normalizations fit independently.

e ObsID 0013140101.

f ObsID 0201830501.

g ObsID 0013140201

h ObsID 0554170201

i ObsID 0554170101.

j Used pile-up model.

presence of XRBs provides a reasonable estimate to decompose the soft X-ray emission in the absence of resolvable nuclear emission.

In the subsequent analysis (i.e., Section 5), we use $L_{x, \mathrm{SF}, \text { Chandra }}$ and $L_{x, \mathrm{AGN}, \text { Chandra }}$ as $L_{x, \mathrm{SF}}$ and $L_{x, \mathrm{AGN}}$ for the 11 Chandra sources with high signal-to-noise. $L_{x, \mathrm{SF}}$ and $L_{x, \mathrm{AGN}}$ for the remaining $24 \mathrm{Sy} 2 \mathrm{~s}$ are estimated via the Monte Carlo simulations outlined above. These values are listed in Tables 8 and 9 for the [O III] and $12 \mu \mathrm{m}$ samples, respectively.

\section{CONSTRAINTS FROM INFRARED DATA}

We compare our X-ray decomposition with information gleaned from high-resolution infrared spectroscopy, using diagnostics that cleanly trace the separate processes of AGN and star formation activity. The flux of the [O IV $] 26 \mu \mathrm{m}$ line has been shown to accurately describe intrinsic AGN flux (Rigby et al. 2009; Diamond-Stanic et al. 2009; Meléndez et al. 2008; LaMassa et al. 2010) while the [Ne II]12.8 $\mu \mathrm{m}$ emission 
Table 5

[O III] Sample Fluxes $\left(10^{-14} \mathrm{erg} \mathrm{cm}^{-2} \mathrm{~s}^{-1}\right)$

\begin{tabular}{lccc}
\hline \hline Galaxy & $F_{0.5-2 \mathrm{keV}}$ & $F_{\text {APEC }}$ & $F_{\text {pow }}$ \\
\hline Mrk 0609 & $79.1_{-3.62}^{+3.62}$ & $4.82_{-2.15}^{+2.14}$ & $74.3_{-2.92}^{+2.92}$ \\
IC 0486 & $35.0_{-8.84}^{+17.6}$ & $0.45_{-0.39}^{+1.12}$ & $34.6_{-8.83}^{+17.6}$ \\
2MASX J08035923+2345201 & $1.24_{-0.71}^{+0.86}$ & $0.83_{-0.70}^{+0.84}$ & $0.41_{-0.14}^{+0.17}$ \\
2MASX J08244333+2959238 & $5.25_{-1.58}^{+1.26}$ & $3.04_{-1.47}^{+1.04}$ & $2.21_{-0.59}^{+0.71}$ \\
2MASX J10181928+3722419 & $2.27_{-0.62}^{+0.92}$ & $1.04_{-0.43}^{+0.80}$ & $1.23_{-0.44}^{+0.46}$ \\
2MASX J11110693+0228477 & $1.44_{-0.59}^{+419}$ & $0.42_{-0.36}^{+419}$ & $1.02_{-0.46}^{+1.31}$ \\
CGCG 242-028 & $1.17_{-0.75}^{+2.52}$ & $0.80_{-0.73}^{+2.50}$ & $0.37_{-0.19}^{+0.33}$ \\
SBS 1133+572 & $3.46_{-0.82}^{+1.27}$ & $0.52_{-0.35}^{+0.36}$ & $2.94_{-0.74}^{+1.22}$ \\
Mrk 1457 & $2.90_{-1.82}^{+10.3}$ & $2.36_{-1.79}^{+10.3}$ & $0.54_{-0.32}^{+0.66}$ \\
2MASX J11570483+5249036 & $3.83_{-0.66}^{+1.31}$ & $0.91_{-0.41}^{+1.09}$ & $2.92_{-0.51}^{+0.72}$ \\
2MASX J12183945+4706275 & $1.54_{-0.54}^{+2.15}$ & $0.55_{-0.31}^{+2.10}$ & $0.99_{-0.44}^{+0.47}$ \\
2MASX J12384342+0927362 & $6.23_{-0.76}^{+2.81}$ & $0.86_{-0.39}^{+2.58}$ & $5.37_{-0.65}^{+1.12}$ \\
NGC 5695 & $2.57_{-0.90}^{+5.37}$ & $0.64_{-0.56}^{+4.31}$ & $1.93_{-0.71}^{+3.20}$ \\
\hline
\end{tabular}

reliably tracks star formation in both quiescent and active systems (LaMassa et al. 2012). We therefore use these fluxes to decompose the soft X-ray emission into a star-forming and an AGN component by solving $L_{0.5-2 \mathrm{keV}}=\alpha \times L_{[\mathrm{Ne} \mathrm{II}]}+\beta \times L_{[\mathrm{O} \text { Iv] }}$ using ordinary least-squares multiple linear regression on both the $12 \mu \mathrm{m}$ and [O III] samples, obtaining $\alpha=1.07$ and $\beta=$ 0.16 (see LaMassa et al. 2010, 2012 for calculated values of $L_{[\mathrm{O} \text { IV] }}$ and $L_{\text {[Ne II] }}$ for these samples). Here $L_{0.5-2 \mathrm{keV}}$ represents the total soft X-ray emission (i.e., Column 1 in Tables 5 and 6).

Figure 2 illustrates the results of this decomposition. We consider the X-ray variable Sy2s, NGC 5506, and NGC 7172 as independent data points, but we do average the soft X-ray flux for the latter two NGC 5506 observations, which are consistent. Though we have only one IR measurement for each of these sources, we do not expect the infrared emission to vary as much as the X-ray emission. We color-code the sources in Figure 2 according to sample: cyan triangles represent the $12 \mu \mathrm{m}$ Sy2s (with the X-ray variable sources connected by a vertical line), blue diamonds denote the subset of $12 \mu \mathrm{m}$ sources with high signal-to-noise Chandra imaging of the unresolved emission, and red squares mark the [O III]-selected sources. The overplotted line indicates where the two quantities are equal. With the exception of a handful of outliers, a relatively good agreement is present between the observed soft X-ray emission and the IR decomposition. We note that this consistency holds for both the $12 \mu \mathrm{m}$ and [O III] samples, with no apparent systematic offset introduced from different sample selection techniques. In the following analysis, we therefore use $\alpha \times L_{[\mathrm{Ne} \text { II] }}$ and $\beta \times L_{\left[\mathrm{O}_{\mathrm{IV}}\right]}$ as an independent estimate of the starburst and AGN contribution, respectively, to the soft X-ray emission.

Table 6

$12 \mu \mathrm{m}$ Sample Fluxes $\left(10^{-14} \mathrm{erg} \mathrm{cm}^{-2} \mathrm{~s}^{-1}\right)$

\begin{tabular}{|c|c|c|c|c|c|}
\hline Galaxy & $F_{0.5-2 \mathrm{keV}}$ & $F_{0.5-2 \mathrm{keV} \text {,extended }}{ }^{\mathrm{a}}$ & $F_{\mathrm{APEC}}$ & $F_{\text {pow }}$ & Comments \\
\hline NGC 0424 & $31.5_{-8.53}^{+12.5}$ & $\ldots$ & $10.6_{-7.81}^{+12.1}$ & $20.9_{-3.43}^{+3.26}$ & \\
\hline NGC 1144 & $8.39_{-1.61}^{+1.25}$ & $\ldots$ & $2.87_{-1.39}^{+0.87}$ & $5.52_{-0.81}^{+0.90}$ & \\
\hline NGC 1320 & $28.4_{-5.46}^{+15.8}$ & $\ldots$ & $10.5_{-4.92}^{+15.6}$ & $17.9_{-2.73}^{+2.38}$ & \\
\hline NGC 1386 & $25.2_{-3.99}^{+6.35}$ & $8.48_{-1.36}^{+1.41}$ & $11.7_{-3.83}^{+6.19}$ & $13.5_{-1.12}^{+1.43}$ & \\
\hline NGC 1667 & $7.31_{-1.52}^{+1.45}$ & $\ldots$ & $3.22_{-1.10}^{+1.03}$ & $4.09_{-1.05}^{+1.01}$ & \\
\hline F05189-2524 & $12.1_{-1.00}^{+2.02}$ & $\ldots$ & $2.22_{-0.68}^{+1.88}$ & $9.88_{-0.74}^{+0.74}$ & \\
\hline NGC 3982 & $9.14_{-0.96}^{+0.90}$ & $7.47_{-2.49}^{+1.96}$ & $3.39_{-0.73}^{+0.63}$ & $5.75_{-0.62}^{+0.64}$ & \\
\hline NGC 4388 & $28.5_{-7.0}^{+16.0}$ & $23.3_{-6.4}^{+6.6}$ & $17.8_{-6.6}^{+15.4}$ & $10.7_{-2.18}^{+3.15}$ & Chandra and XMM-Newton observations averaged \\
\hline NGC 4501 & $7.24_{-1.30}^{+1.57}$ & $5.47_{-1.47}^{+1.42}$ & $3.83_{-0.95}^{+1.06}$ & $3.41_{-0.88}^{+1.15}$ & \\
\hline TOLOLO 1238-364 & $14.2_{4.2}^{+49}$ & $\ldots$ & $5.43_{-3.80}^{+5.01}$ & $8.77_{-1.82}^{+49}$ & \\
\hline NGC 4968 & $5.85_{-1.05}^{+1.14}$ & $\cdots$ & $1.27_{-0.60}^{+0.67}$ & $4.58_{-0.86}^{+0.93}$ & \\
\hline M-3-34-64 & $50.8_{-7.81}^{+6.37}$ & $\cdots$ & $33.4_{-7.29}^{+4.72}$ & $17.4_{-2.80}^{+4.28}$ & \\
\hline NGC 5135 & $34.5_{-1.68}^{+1.66}$ & $16.4_{-5.9}^{+7.1}$ & $17.0_{-1.27}^{+1.23}$ & $17.5_{-1.10}^{+1.12}$ & \\
\hline NGC 5194 & $69.6_{-6.07}^{+6.82}$ & $56.1_{-7.9}^{+8.0}$ & $52.7_{-5.97}^{+6.71}$ & $16.9_{-1.09}^{+1.19}$ & \\
\hline NGC 5347 & $2.94_{-0.46}^{+0.87}$ & $\cdots$ & $0.50_{-0.30}^{+0.80}$ & $2.44_{-0.35}^{+0.34}$ & \\
\hline Mrk 463 & $14.3_{-2.72}^{+2.68}$ & $6.04_{-1.88}^{+9.40}$ & $7.50_{-2.62}^{+2.59}$ & $6.80_{-0.73}^{+0.70}$ & \\
\hline NGC 5506 & $308_{-53}^{+56}$ & $\cdots$ & $6.07_{-4.55}^{+4.48}$ & $302_{-53}^{+56}$ & ObsIDs $0013140101,0201830201,0201830301,0201830401$ \\
\hline NGC 5506 & $444_{-37}^{+37}$ & $\ldots$ & 0 & $444_{-37}^{+37}$ & ObsIDs 0013140201 and 0201830501 \\
\hline NGC 5506 & $457_{-43}^{+37}$ & $\cdots$ & $10.5_{-1.52}^{+1.65}$ & $446_{-43}^{+37}$ & ObsIDs 0554170201 and 0554170101 \\
\hline Arp 220 & $4.90_{-1.34}^{+1.19}$ & $4.38_{-0.77}^{+1.11}$ & $3.05_{-1.24}^{+0.95}$ & $1.85_{-0.51}^{+0.71}$ & \\
\hline NGC 6890 & $11.5_{-3.76}^{+6.19}$ & $\cdots$ & $2.62_{-1.40}^{+1.64}$ & $8.88_{-3.49}^{+5.97}$ & \\
\hline IC 5063 & $22.9_{-3.18}^{+3.12}$ & $4.79_{-1.20}^{+1.15}$ & $4.57_{-1.58}^{+1.58}$ & $18.3_{-2.76}^{+2.69}$ & \\
\hline NGC 7130 & $23.6_{-1.89}^{+2.63}$ & $8.55_{-1.20}^{+3.65}$ & $10.3_{-0.96}^{+1.24}$ & $13.3_{-1.62}^{+2.32}$ & \\
\hline NGC 7172 & $20.3_{-2.45}^{+2.28}$ & $\ldots$ & $2.05_{-0.51}^{+0.50}$ & $18.3_{-2.40}^{+2.22}$ & ObsID 0414580101 \\
\hline NGC 7172 & $11.8_{-0.72}^{+0.75}$ & $\ldots$ & $2.06_{-0.28}^{+0.27}$ & $9.74_{-0.67}^{+0.70}$ & ObsIDs 0147920601 and 0202860101 \\
\hline NGC 7582 & $40.3_{-3.7}^{+3.2}$ & $30.8_{-2.8}^{+3.2}$ & $15.2_{-3.1}^{+2.5}$ & $25.1_{-2.0}^{+2.0}$ & Chandra and XMM-Newton observations averaged \\
\hline NGC 7674 & $17.5_{-4.95}^{+2.91}$ & $\cdots$ & $13.6_{-4.74}^{+2.17}$ & $3.90_{-1.42}^{+1.94}$ & \\
\hline
\end{tabular}

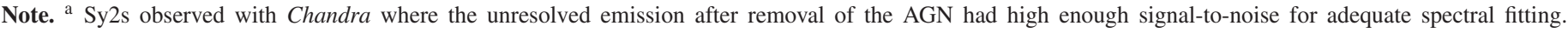
$F_{0.5-2 \mathrm{keV} \text {,extended }}$ flux corresponds to this extended emission from the host galaxy (i.e., with the AGN removed). 


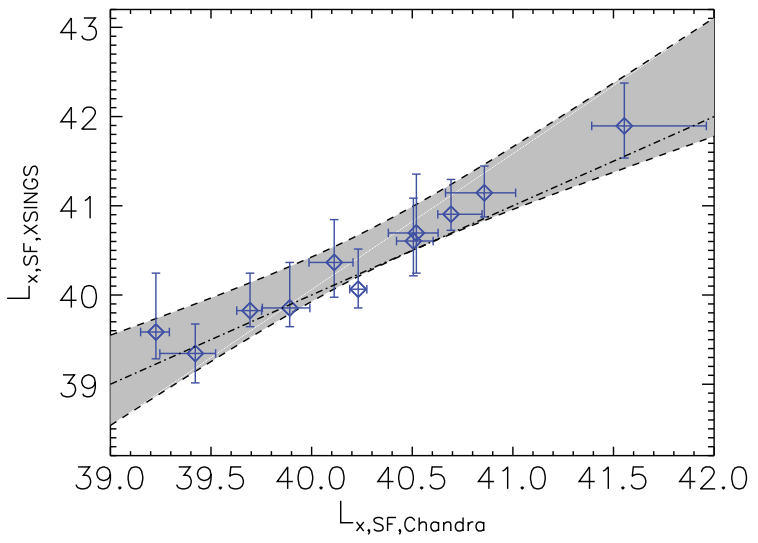

(a)

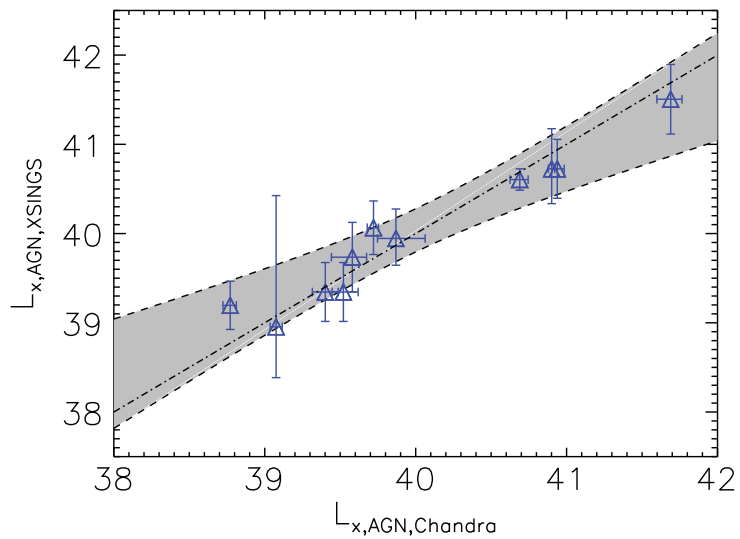

(b)

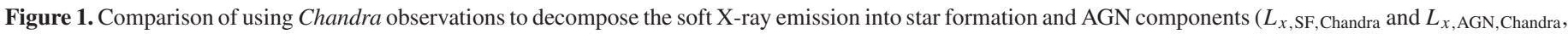

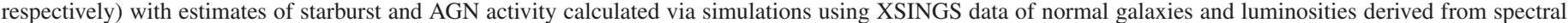

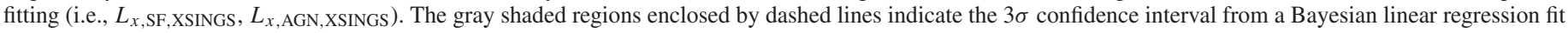

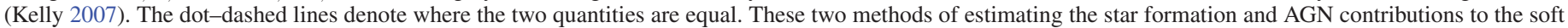
$\mathrm{X}$-ray emission are consistent.

(A color version of this figure is available in the online journal.)

Table 7

Chandra Spectral Fits to Unresolved Emission Only ${ }^{\mathrm{a}}$

\begin{tabular}{|c|c|c|c|c|c|c|c|}
\hline$\overline{\text { Galaxy }}$ & $\begin{array}{c}N_{\mathrm{H}, 1} \\
\left(10^{22} \mathrm{~cm}^{-2}\right)\end{array}$ & $\begin{array}{c}k T_{1} \\
(\mathrm{keV})\end{array}$ & $\begin{array}{c}k T_{2} \\
(\mathrm{keV})\end{array}$ & $\Gamma$ & $\begin{array}{c}N_{\mathrm{H}, 2} \\
\left(10^{22} \mathrm{~cm}^{-2}\right)\end{array}$ & $\begin{array}{c}\chi^{2} 2 \text { APECs } \\
\text { DOF }\end{array}$ & $\begin{array}{c}\chi^{2} 1 \text { APEC } \\
\text { DOF }\end{array}$ \\
\hline NGC 1386 & $<0.05$ & $0.58_{-0.13}^{+0.09}$ & $\ldots$ & $3.23_{-0.34}^{+0.38}$ & $\begin{array}{l}48.9_{-12.4}^{+32.0} \\
\end{array}$ & $\ldots$ & $79.0(54)$ \\
\hline NGC $3982^{b}$ & 0.01 & $0.20_{-0.06}^{+0.06}$ & $\ldots$ & $2.04_{-0.68}^{+0.68}$ & $\ldots$ & $\cdots$ & $46.5(25)$ \\
\hline NGC $4388^{b}$ & 0.03 & $0.60_{-0.04}^{+0.04}$ & $0.16_{-0.02}^{+0.04}$ & $0.98_{-0.50}^{+0.51}$ & $\ldots$ & $85.4(83)$ & $123(84)$ \\
\hline NGC $4501^{b}$ & 0.03 & $0.37_{-0.07}^{+0.22}$ & $\cdots$ & $2.26_{-0.50}^{+0.44}$ & $\ldots$ & $\ldots$ & $31.2(38)$ \\
\hline NGC 5135 & $<0.13$ & $0.84_{-0.09}^{+0.09}$ & $0.39_{-0.09}^{+0.11}$ & $2.24_{-0.31}^{+0.33}$ & $\ldots$ & $62.8(60)$ & $77.9(62)$ \\
\hline NGC $5194^{b}$ & 0.02 & $0.60_{-0.01}^{+0.01}$ & $0.18_{-0.02}^{+0.03}$ & $3.13_{-0.09}^{+0.10}$ & $7.92_{-1.08}^{+1.38}$ & $525(352)$ & $616(353)$ \\
\hline Mrk 463 & $<0.05$ & $0.62_{-0.07}^{+0.05}$ & $\cdots$ & $2.50_{-0.47}^{+0.75}$ & $34.6_{-7.7}^{+10.5}$ & $\cdots$ & $86(71)$ \\
\hline $\operatorname{Arp} 220^{\mathrm{b}}$ & $<0.04$ & $0.73_{-0.12}^{+0.07}$ & $\ldots$ & $2.10_{-0.36}^{+0.44}$ & $\ldots$ & $\ldots$ & $69.3(57)$ \\
\hline IC $5063^{b}$ & 0.06 & $0.34_{-0.04}^{+0.09}$ & $\ldots$ & $1.51_{-0.59}^{+0.55}$ & $28.5_{-5.0}^{+6.2}$ & $\ldots$ & $71.5(57)$ \\
\hline NGC 7130 & $0.05_{-0.03}^{+0.07}$ & $0.47_{-0.10}^{+0.07}$ & $\ldots$ & $2.24_{-0.29}^{+0.37}$ & $\ldots$ & $\ldots$ & $58.2(50)$ \\
\hline NGC 7582 & $0.03_{-0.02}^{+0.02}$ & $0.73_{-0.05}^{+0.04}$ & $\ldots$ & $2.37_{-0.20}^{+0.23}$ & $41.8_{-8.2}^{+10.1}$ & $\cdots$ & $142(128)$ \\
\hline
\end{tabular}

Notes.

a All abundances frozen to solar.

${ }^{\mathrm{b}}$ Best-fit absorption same as Galactic absorption. This parameter was then frozen to the Galactic value.

Table 8

[O III] Sample Star Formation and AGN Luminosities

\begin{tabular}{lcc}
\hline \hline Galaxy & $\log \left(L_{x, \mathrm{SF}}\right)$ & $\log \left(L_{x, \mathrm{AGN}}\right)$ \\
\hline Mrk 0609 & $41.38_{-0.39}^{+0.54}$ & $42.28_{-0.06}^{+0.06}$ \\
IC 0486 & $40.39_{-0.42}^{+0.87}$ & $41.83_{-0.24}^{+0.27}$ \\
2MASX J08035923+2345201 & $40.45_{-0.33}^{+0.87}$ & $39.64_{-0.30}^{+0.33}$ \\
2MASX J08244333+2959238 & $40.81_{-0.33}^{+0.60}$ & $40.18_{-0.30}^{+0.30}$ \\
2MASX J10181928+3722419 & $40.99_{-0.36}^{+0.72}$ & $40.54_{-0.24}^{+0.27}$ \\
SBS 1133+572 & $40.76_{-0.33}^{+0.78}$ & $41.17_{-0.15}^{+0.15}$ \\
Mrk 1457 & $41.74_{-0.42}^{+0.96}$ & $40.15_{-0.48}^{+0.57}$ \\
2MASX J11570483+5249036 & $40.67_{-0.36}^{+0.84}$ & $40.81_{-0.12}^{+0.12}$ \\
2MASX J12183945+4706275 & $41.68_{-0.42}^{+0.93}$ & $41.20_{-0.30}^{+0.33}$ \\
2MASX J12384342+0927362 & $41.69_{-0.42}^{+0.87}$ & $41.90_{-0.12}^{+0.15}$ \\
NGC 5695 & $40.78_{-0.81}^{+0.96}$ & $39.79_{-0.41}^{+0.42}$ \\
\hline
\end{tabular}

\section{RESULTS: COMPARISON OF SOFT X-RAY DECOMPOSITION WITH IR}

We plot $L_{x, \mathrm{SF}}$ as a function of $\alpha \times L_{\text {[Ne II] }}$ and $L_{x, \mathrm{AGN}}$ as a function of $\beta \times L_{[\mathrm{O} \text { Iv] }}$ in Figure 3. We note that the abscissa errors are on the order of the symbol size, due to the $5 \sigma$ detection limit we imposed on IR detections (see LaMassa et al. 2012), and therefore are not plotted in Figure 3, though they are included in the errors for the Bayesian linear regression routine. Due to the large error on $L_{x, \mathrm{AGN}}$ for $\mathrm{NGC} 7674$, this source was dropped from the $L_{x, \mathrm{AGN}}$ versus $\beta \times L_{[\mathrm{O} \text { Iv }]}$ analysis.

In Figure 3, a dot-dashed line is plotted to indicate where the two quantities are equal while the gray shaded region illustrates the $3 \sigma$ confidence interval on the regression line. $L_{x, \mathrm{SF}}$ and $L_{x, \mathrm{AGN}}$ are approximately equivalent to $\alpha \times L_{\text {[Ne II] }}$ and $\beta \times L_{\left[\mathrm{O}_{\mathrm{IV}}\right]}$, respectively, albeit with wide scatter for a handful of individual sources. Deviations of the regression fit from equality at greater than the $3 \sigma$ level are slight, and appear at higher luminosities for the star formation decomposition $\left(>10^{41} \mathrm{erg} \mathrm{s}^{-1} \mathrm{~cm}^{-2}\right.$ ) and moderate luminosities for the AGN 
Table 9

$12 \mu \mathrm{m}$ Sample Star Formation and AGN Luminosities

\begin{tabular}{|c|c|c|c|}
\hline Galaxy & $\log \left(L_{x, \mathrm{SF}}\right)$ & $\log \left(L_{x, \mathrm{AGN}}\right)$ & Comments \\
\hline NGC 0424 & $40.99_{-0.54}^{+0.69}$ & $40.72_{-0.18}^{+0.21}$ & \\
\hline NGC 1144 & $40.78_{-0.24}^{+0.66}$ & $40.87_{-0.21}^{+0.21}$ & \\
\hline NGC 1320 & $40.33_{-0.51}^{+1.05}$ & $40.42_{-0.21}^{+0.21}$ & \\
\hline NGC $1386^{\mathrm{a}}$ & $39.23_{-0.08}^{+0.07}$ & $39.52_{-0.07}^{+0.10}$ & \\
\hline NGC 1667 & $40.30_{-0.21}^{+0.60}$ & $40.06_{-0.24}^{+0.24}$ & \\
\hline F05189-2524 & $41.20_{-0.30}^{+0.78}$ & $41.56_{-0.12}^{+0.15}$ & \\
\hline NGC $3982^{a}$ & $39.42_{-0.18}^{+0.10}$ & $38.77_{-0.05}^{+0.04}$ & \\
\hline NGC $4388^{a}$ & $40.52_{-0.14}^{+0.11}$ & $39.87_{-0.12}^{+0.19}$ & \\
\hline NGC $4501^{\mathrm{a}}$ & $39.89_{-0.14}^{+0.10}$ & $39.40_{-0.09}^{+0.09}$ & \\
\hline TOLOLO 1238-364 & $40.63_{-0.57}^{+0.72}$ & $40.31_{-0.45}^{+0.48}$ & \\
\hline NGC 4968 & $39.67_{-0.33}^{+0.69}$ & $39.88_{-0.12}^{+0.12}$ & \\
\hline M-3-34-64 & $41.47_{-0.21}^{+0.45}$ & $40.84_{-0.60}^{+0.60}$ & \\
\hline NGC $5135^{\mathrm{a}}$ & $40.86_{-0.19}^{+0.16}$ & $40.90_{-0.02}^{+0.02}$ & \\
\hline NGC $5194^{\mathrm{a}}$ & $39.69_{-0.07}^{+0.06}$ & $39.08_{-0.04}^{+0.04}$ & \\
\hline NGC 5347 & $39.34_{-0.48}^{+0.84}$ & $39.47_{-0.12}^{+0.15}$ & \\
\hline $\operatorname{Mrk} 463^{\mathrm{a}}$ & $41.55_{-0.16}^{+0.41}$ & $41.69_{-0.09}^{+0.07}$ & \\
\hline NGC 5506 & $39.88_{-0.27}^{+0.93}$ & $41.38_{-0.09}^{+0.12}$ & ObsIDs 0013140101, 0201830201, 0201830301, 0201830401 \\
\hline NGC 5506 & 0 & $41.53_{-0.06}^{+0.09}$ & ObsIDs 0013140201 and 0201830501 \\
\hline NGC 5506 & $40.06_{-0.21}^{+0.45}$ & $41.53_{-0.06}^{+0.06}$ & ObsIDs 0554170201 and 0554170101 \\
\hline $\operatorname{Arp} 220^{\mathrm{a}}$ & $40.51_{-0.08}^{+0.10}$ & $39.58_{-0.14}^{+0.09}$ & \\
\hline NGC 6890 & $39.83_{-0.39}^{+0.69}$ & $39.94_{-0.24}^{+0.24}$ & \\
\hline IC $5063^{\mathrm{a}}$ & $40.11_{-0.13}^{+0.09}$ & $40.69_{-0.06}^{+0.06}$ & \\
\hline NGC $7130^{\mathrm{a}}$ & $40.69_{-0.07}^{+0.15}$ & $40.94_{-0.04}^{+0.05}$ & \\
\hline NGC 7172 & $39.85_{-0.36}^{+0.39}$ & $40.45_{-0.06}^{+0.06}$ & ObsID 0414580101 \\
\hline NGC 7172 & $39.67_{-0.15}^{+0.45}$ & $40.18_{-0.12}^{+0.12}$ & ObsIDs 0147920601 and 0202860101 \\
\hline NGC $7582^{\mathrm{a}}$ & $40.23_{-0.04}^{+0.04}$ & $39.72_{-0.04}^{+0.03}$ & \\
\hline NGC 7674 & $41.53_{-0.21}^{+0.51}$ & $40.03_{-0.39}^{+2.70}$ & \\
\hline
\end{tabular}

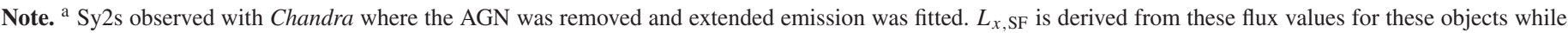
$L_{x, \mathrm{AGN}}$ is simply $L_{0.5-2 \mathrm{keV}}-L_{x, \mathrm{SF}}$. For the remaining sources, $L_{x, \mathrm{SF}}$ and $L_{x}$, AGN are derived as described in the text.

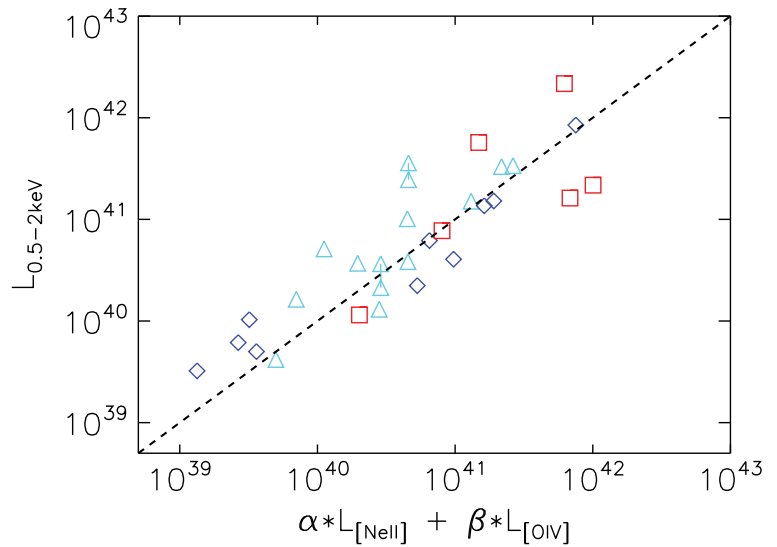

Figure 2. Results of decomposing the soft X-ray flux $(0.5-2 \mathrm{keV})$ into a star formation and AGN components, using the luminosity of the [Ne II] line as a proxy for the former and the luminosity of the [O IV] line to parameterize the latter. The constants $\alpha$ and $\beta$ were calculated using ordinary least-squares multiple linear regression, where we derive $\alpha=1.07$ and $\beta=0.16$. The overplotted line indicates where the two quantities are equal. Cyan triangles represent the $12 \mu \mathrm{m} \mathrm{Sy} 2 \mathrm{~s}$ (with the X-ray variable sources connected by a vertical line), blue diamonds denote the subset of $12 \mu \mathrm{m}$ sources with high signal-to-noise Chandra imaging of the unresolved emission, and red squares mark the [O III] selected sources.

(A color version of this figure is available in the online journal.) parameterization $\left(10^{39} \lesssim L_{x, \text { AGN }} \lesssim 5 \times 10^{41} \mathrm{erg} \mathrm{s}^{-1} \mathrm{~cm}^{-2}\right)$. As mentioned previously, the NLR for IC 5063 takes up a significant fraction of the $\mathrm{X}$-ray extraction region, where contributions from resolved NLR emission could potentially bias the X-ray estimate of host galaxy star formation. However, as seen in Figure 3, where IC 5068 is the blue diamond at $\alpha \times L_{[\mathrm{Ne} \text { II] }}=40.4 \mathrm{dex}$ and $L_{x, \mathrm{SF}}=40.1 \mathrm{dex}$, this source lies well within the shaded region. Hence NLR contamination appears to be negligible, possibly due to the removal of the AGN from the Chandra imaging analysis.

We note that aperture bias can contribute to these slight disagreements: [Ne II] ([O IV]) is measured through the ShortHigh (Long-High) module on the Infrared Spectrograph on Spitzer, corresponding to sizes $4^{\prime \prime} .7 \times 11^{\prime \prime} .3\left(11^{\prime \prime} .1 \times 22^{\prime \prime} .3\right)$, whereas the X-ray apertures capture the full emission from the host galaxy (see Tables 1 and 2). Hence, biases can be introduced from comparing parameters that sample different physical scales of the host galaxy, introducing scatter into the relation. In the AGN parameterization, there are four obvious outliers from the relation: IC 0486 and Mrk 463 (members of the [O III] sample with overpredicted $L_{x, \mathrm{AGN}}$, red squares in Figure 3), NGC 4388 (from the $12 \mu \mathrm{m}$ sample with underpredicted $L_{x, \mathrm{AGN}}$, blue diamonds in Figure 3 ), and NGC 5506 (from the $12 \mu \mathrm{m}$ sample with overpredicted $L_{x \text {, AGN }}$, an X-ray variable source with a vertical line connecting the cyan 


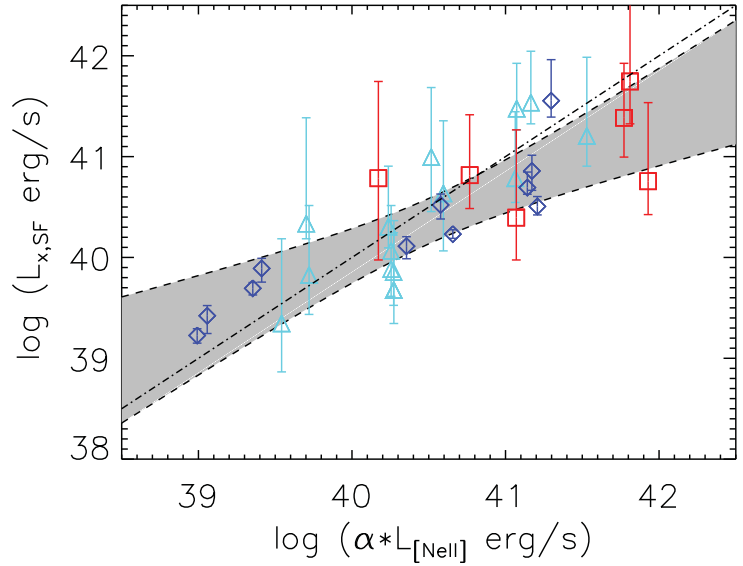

(a)

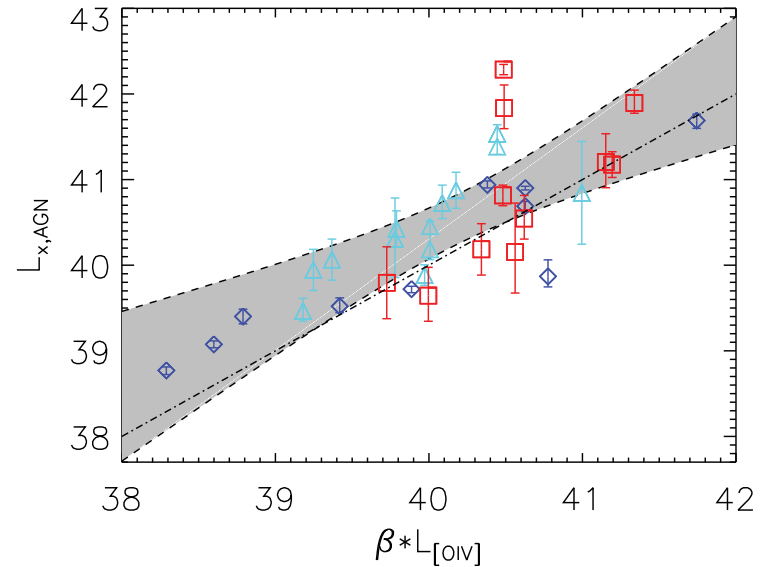

(b)

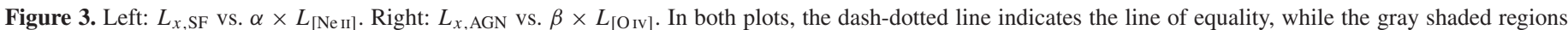

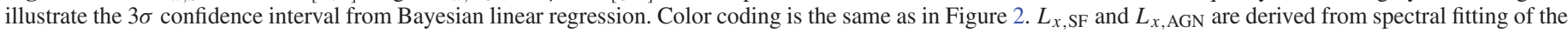

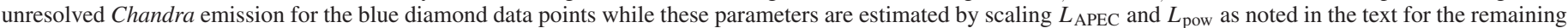

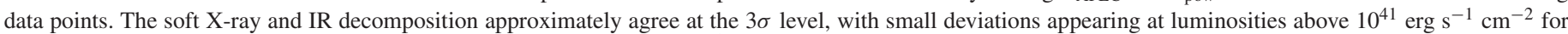
the star formation parameterization and between $10^{39} \lesssim L_{x}$, AGN $\lesssim 5 \times 10^{41} \mathrm{erg} \mathrm{s}^{-1} \mathrm{~cm}^{-2}$ for the AGN decomposition.

(A color version of this figure is available in the online journal.)

triangles in Figure 3). Interestingly, there are no correlations between outliers in the $L_{x, \mathrm{AGN}}$ parameterization and physical size of X-ray emitting region (where mismatch between aperture sizes between Chandra or XMM-Newton and Spitzer could introduce disagreements), quality of soft X-ray spectra, sample selection, source variability, or even method for estimating $L_{x, \mathrm{AGN}}$. In general, however, deviations of the best-fit trends between $L_{x, \mathrm{SF}}$ versus $\alpha \times L_{[\mathrm{Ne} \text { I] }}$ and $L_{x, \mathrm{AGN}}$ versus $\beta \times L_{[\mathrm{O} \text { Iv] }}$ from equality at the $3 \sigma$ level are slight. Such an agreement demonstrates that our method of using low-resolution CCD spectroscopy to decompose the soft X-ray emission into a starforming and an AGN component is moderately successful, with greater consistency in the star formation estimation. $L_{x, \mathrm{SF}}$ can then be used to estimate the host galaxy SFR in Sy2s using the $L_{0.5-2 \mathrm{keV}}$ calibrations from Ranalli et al. (2003) and PereiraSantaella et al. (2011).

\subsection{Comparison with Far-infrared Derived SFRs}

For the $12 \mu \mathrm{m}$ sample, we have tested the efficacy of our decomposition by deriving a soft X-ray SFR using the PereiraSantaella et al. (2011) calibration and comparing this with the far-infrared (FIR) SFR using the Kennicutt (1998) calibration, using IRAS flux densities reported in Spinoglio \& Malkan (1989). The [O III] sample, however, lacks a reliable independent host galaxy wide SFR. These sources were not detected by IRAS and the SDSS SFRs derived through the $3^{\prime \prime}$ fiber (see LaMassa et al. 2012 for a discussion) only covers the innermost regions of the galaxy, while the X-ray aperture captures the full emission. We therefore use only the $12 \mu \mathrm{m}$ sample to test the X-rayderived SFRs.

To estimate SFR $_{\text {FIR }}$, we used the sum of $60 \mu \mathrm{m}$ and $100 \mu \mathrm{m}$ IRAS flux densities. We note that the Kennicutt (1998) calibration is based on MIR through FIR luminosities for starburst galaxies, and in such systems, including the IRAS $12 \mu \mathrm{m}$ and $25 \mu \mathrm{m}$ luminosities as part of the FIR luminosity is important. However, these MIR luminosities have significant contributions from dust heated by the AGN, so we only consider the $60 \mu \mathrm{m}$ and $100 \mu \mathrm{m}$ luminosities when deriving $\mathrm{SFR}_{\mathrm{FIR}}$, which are less contaminated by the AGN. As shown in Figure 4, a good agreement exists between $\mathrm{SFR}_{0.5-2 \mathrm{kev}}$ and $\mathrm{SFR}_{\mathrm{FIR}}$, despite the

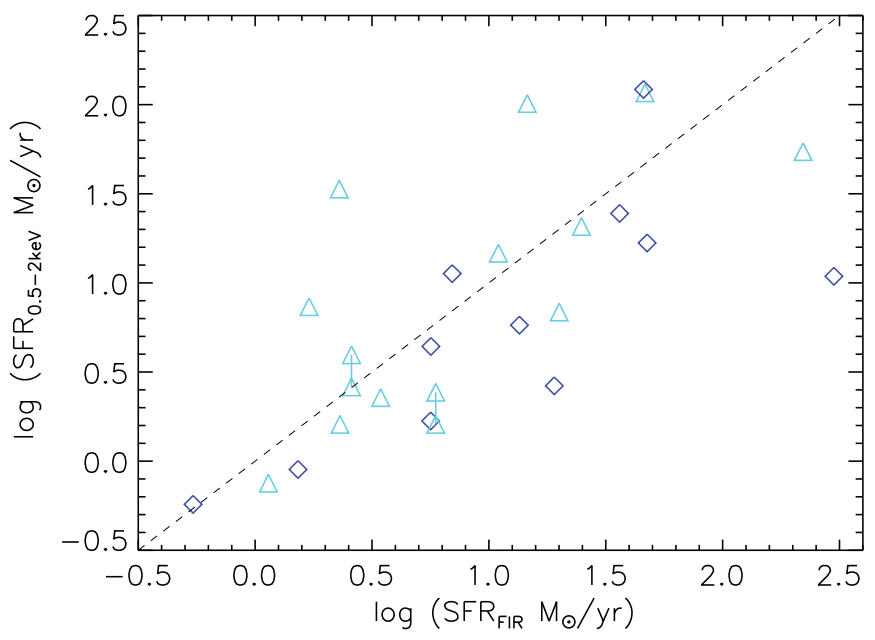

Figure 4. X-ray-derived SFRs ( $\mathrm{SFR}_{0.5-2 \mathrm{keV}}$ ) using our estimate of $L_{x, \mathrm{SF}}$ and the calibration of Pereira-Santaella et al. (2011) as a function of FIR-derived SFRs (SFR ${ }_{\mathrm{FIR}}$ ), using IRAS $60 \mu \mathrm{m}$ and $100 \mu \mathrm{m}$ luminosities and Kennicutt's (1998) calibration, for the $12 \mu \mathrm{m}$ sample. The overplotted dashed line shows where the two SFRs are equal. A relatively good agreement exists, with the two most significant outliers with the largest SFR FIR values (Arp 220 and F05189-2524) being ULIRGs.

(A color version of this figure is available in the online journal.)

latter SFR parameterization being most appropriate for starburst galaxies. This agreement suggests that any unresolved photoionized emission from the NLR does not introduce any systematic offsets into our results, but rather operates at a level less than the observed scatter. The two Sy2s that are the greatest outliers (i.e.,

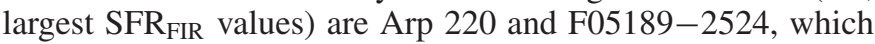
are ultra luminous infrared galaxies (ULIRGs).

\section{CONCLUSION}

We have modeled the $0.5-10 \mathrm{keV}$ spectra of two homogeneous samples of Seyfert 2 galaxies to disentangle the starburst and AGN emission in soft X-rays $(0.5-2 \mathrm{keV})$. Eleven of these Sy2s, observed with Chandra, had high signal-to-noise unresolved emission after removing the AGN. We derive $L_{x, \mathrm{SF}}$ from 
spectrally fitting this unresolved emission and assign $L_{x, \text { AGN }}$ to the difference between the total soft X-ray emission and $L_{x}$,SF.

The remaining 24 sources were decomposed into AGN and star formation components by modeling the soft X-ray emission with a power law and thermal model. The luminosities of these sources were converted to $L_{x, \mathrm{SF}}$ and $L_{x, \mathrm{AGN}}$ values based on a scaling factor derived from XSINGS analysis on normal starforming galaxies. To account for errors on the luminosities derived from spectral fits and the scaling factor, we executed Monte Carlo simulations, assuming a Gaussian distribution of random variables centered on the best-fit values of $L_{\mathrm{APEC}}, L_{\mathrm{pow}}$, and $R$, with the spread corresponding to the errors on these parameters. Using Equations (4) and (5), we calculate $L_{x, \mathrm{SF}}$ and $L_{x, \text { AGN }}$ from the 1000 simulated parameters and use the mode of this distribution as our estimate of the X-ray luminosities due to star formation and AGN activity.

Our conclusions are summarized as follows.

1. The soft X-ray spectra of $10 \mathrm{Sy} 2 \mathrm{~s}$ were well fitted by two thermal model components, indicating the presence of a two-temperature gas. This result is similar to what has been observed in starburst galaxies (Dahlem et al. 1998; Strickland et al. 2004).

2. For the subset of $11 \mathrm{Sy} 2 \mathrm{~s}$ with high signal-to-noise Chandra imaging of unresolved host galaxy emission, estimates of the soft X-ray emission due to star formation and AGNs from both Chandra imaging analysis and from the Monte Carlo simulations agree at the $3 \sigma$ level. In the absence of resolved nuclear emission, scaling $L_{\text {pow }}$ and $L_{\mathrm{AGN}}$ by the factors derived from XSINGS analysis is thus a reasonable method to estimate the AGN and star formation contributions to the soft X-ray emission.

3. The independent decompositions of the soft X-ray luminosity into star formation and AGN components using IR data as a proxy and scaling $L_{\mathrm{APEC}}$ and $L_{\text {pow }}$ based on XSINGS galaxies largely agree within a $3 \sigma$ confidence interval. Deviations of the best-fit regression line from this agreement are slight and appear at higher luminosities $\left(>10^{41} \mathrm{erg} \mathrm{s}^{-1} \mathrm{~cm}^{-2}\right)$ in the star formation decomposition and at moderate luminosities $\left(10^{39} \lesssim L \lesssim\right.$ $5 \times 10^{41} \mathrm{erg} \mathrm{s}^{-1} \mathrm{~cm}^{-2}$ ) when describing AGN emission. Though more scatter in individual sources is evident in the AGN decomposition, the star formation relationship is more consistent among both methods.

4. Comparison of our calculated X-ray SFR using the PereiraSantaella et al. (2011) calibration with an FIR-derived SFR from Kennicutt (1998) for the $12 \mu \mathrm{m}$ sample shows general agreement.

We have demonstrated that analysis of low-resolution CCD $\mathrm{X}$-ray spectra can effectively disentangle emission from AGN activity and star formation in $0.5-2 \mathrm{keV} \mathrm{X}$-rays. Using the decomposition we have presented, $L_{x}$,SF can be used to cleanly estimate the SFR in Sy2s using existing calibrations (e.g., Ranalli et al. 2003; Pereira-Santaella et al. 2011), complementing previous studies in the optical and infrared and providing a more panchromatic view of the interplay between SMBH accretion and star formation.

This work is funded by ADAP grant number 10-ADAP100167. We thank the anonymous referee for comments and suggestions that improved the manuscript. Funding for the SDSS and SDSS-II has been provided by the Alfred P. Sloan Foundation, the Participating Institutions, the National Science Foundation, the U.S. Department of Energy, the National
Aeronautics and Space Administration, the Japanese Monbukagakusho, the Max Planck Society, and the Higher Education Funding Council for England. The SDSS Web Site is http://www.sdss.org/. The SDSS is managed by the Astrophysical Research Consortium for the Participating Institutions. The Participating Institutions are the American Museum of Natural History, Astrophysical Institute Potsdam, University of Basel, University of Cambridge, Case Western Reserve University, University of Chicago, Drexel University, Fermilab, the Institute for Advanced Study, the Japan Participation Group, Johns Hopkins University, the Joint Institute for Nuclear Astrophysics, the Kavli Institute for Particle Astrophysics and Cosmology, the Korean Scientist Group, the Chinese Academy of Sciences (LAMOST), Los Alamos National Laboratory, the Max-PlanckInstitute for Astronomy (MPIA), the Max-Planck-Institute for Astrophysics (MPA), New Mexico State University, Ohio State University, University of Pittsburgh, University of Portsmouth, Princeton University, the United States Naval Observatory, and the University of Washington.

\section{REFERENCES}

Adelman-McCarthy, J. K., Agüeros, M. A., Allam, S. S., et al. 2006, ApJS, 162,38

Baldwin, J. A., Phillips, M. M., \& Terlevich, R. 1981, PASP, 93, 5

Buchanan, C. L., Gallimore, J. F., O’Dea, C. P., et al. 2006, AJ, 132, 401

Cid Fernandes, R., Heckman, T., Schmitt, H., González Delgado, R. M., \& Storchi-Bergmann, T. 2001, ApJ, 558, 81

Cisternas, M., Jahnke, K., Inskip, K. J., et al. 2011, ApJ, 726, 57

Colbert, E. J. M., Heckman, T. M., Ptak, A. F., Strickland, D. K., \& Weaver, K. A. 2004, ApJ, 602, 231

Dahlem, M., Weaver, K. A., \& Heckman, T. M. 1998, ApJS, 118, 401

Diamond-Stanic, A. M., Rieke, G. H., \& Rigby, J. R. 2009, ApJ, 698, 623

Fabbiano, G. 2006, ARA\&A, 44, 323

Ferrarese, L., \& Merritt, D. 2000, ApJ, 539, L9

Gebhardt, K., Bender, R., Bower, G., et al. 2000, ApJ, 539, L13

Genzel, R., Lutz, D., Sturm, E., et al. 1998, ApJ, 498, 579

Häring, N., \& Rix, H.-W. 2004, ApJ, 604, L89

Ho, L. C., \& Keto, E. 2007, ApJ, 658, 314

Hopkins, P. F., Hernquist, L., Cox, T. J., \& Kereš, D. 2008, ApJS, 175, 356 Kauffmann, G., Heckman, T. M., Tremonti, C., et al. 2003, MNRAS, 346,1055

Kelly, B. C. 2007, ApJ, 665, 1489

Kennicutt, R. C., Jr. 1998, ARA\&A, 36, 189

Kennicutt, R. C., Jr., Armus, L., Bendo, G., et al. 2003, PASP, 115, 928

Kewley, L. J., Dopita, M. A., Sutherland, R. S., Heisler, C. A., \& Trevena, J. 2001, ApJ, 556, 121

Kewley, L. J., Groves, B., Kauffmann, G., \& Heckman, T. 2006, MNRAS, 372,961

Kinkhabwala, A., Sako, M., Behar, E., et al. 2002, ApJ, 575, 732

Kormendy, J., \& Kennicutt, R. C., Jr. 2004, ARA\&A, 42, 603

Kormendy, J., \& Richstone, D. 1995, ARA\&A, 33, 581

LaMassa, S. M., Heckman, T. M., Ptak, A., et al. 2009, ApJ, 705, 568

LaMassa, S. M., Heckman, T. M., Ptak, A., et al. 2010, ApJ, 720, 786

LaMassa, S. M., Heckman, T. M., Ptak, A., et al. 2011, ApJ, 729, 52

LaMassa, S. M., Heckman, T. M., Ptak, A., et al. 2012, ApJ, 758, 1

Lehmer, B. D., Alexander, D. M., Bauer, F. E., et al. 2010, ApJ, 724, 559

Levenson, N. A., Weaver, K. A., Heckman, T. M., Awaki, H., \& Terashima, Y. 2004, ApJ, 602, 135

Levenson, N. A., Weaver, K. A., Heckman, T. M., Awaki, H., \& Terashima, Y. 2005, ApJ, 618, 167

Liedahl, D. A., \& Paerels, F. 1996, ApJ, 468, L33

Magorrian, J., Tremaine, S., Richstone, D., et al. 1998, AJ, 115, 2285

Meléndez, M., Kraemer, S. B., Armentrout, B. K., et al. 2008, ApJ, 682, 94

O’Dowd, M. J., Schiminovich, D., Johnson, B. D., et al. 2009, ApJ, 705,885

Pereira-Santaella, M., Alonso-Herrero, A., Santos-Lleo, M., et al. 2011, A\&A, 535, A93

Persic, M., \& Rephaeli, Y. 2007, A\&A, 463, 481

Persic, M., Rephaeli, Y., Braito, V., et al. 2004, A\&A, 419, 849

Porquet, D., \& Dubau, J. 2000, A\&AS, 143, 495 
Ptak, A., \& Griffiths, R. 2003, in ASP Conf. Ser. 295, Astronomical Data Analysis Software and Systems XII, ed. H. E. Payne, R. I. Jedrzejewski, \& R. N. Hook (San Francisco, CA: ASP), 465

Ranalli, P., Comastri, A., \& Setti, G. 2003, A\&A, 399, 39

Rigby, J. R., Diamond-Stanic, A. M., \& Aniano, G. 2009, ApJ, 700, 1878

Rosa González, D., Terlevich, E., Jiménez Bailón, E., et al. 2009, MNRAS, 399,487

Sanders, D. B., Soifer, B. T., Elias, J. H., et al. 1988, ApJ, 325, 74

Schawinski, K., Treister, E., Urry, C. M., et al. 2011, ApJ, 727, L31
Schmitt, H. R., Donley, J. L., Antonucci, R. R. J., Hutchings, J. B., \& Kinney, A. L. 2003a, ApJS, 148, 327

Schmitt, H. R., Donley, J. L., Antonucci, R. R. J., et al. 2003b, ApJ, 597,768

Spinoglio, L., \& Malkan, M. A. 1989, ApJ, 342, 83

Strickland, D. K., Heckman, T. M., Colbert, E. J. M., Hoopes, C. G., \& Weaver, K. A. 2004, ApJS, 151, 193

Tremaine, S., Gebhardt, K., Bender, R., et al. 2002, ApJ, 574, 740

Treyer, M., Schiminovich, D., Johnson, B. D., et al. 2010, ApJ, 719, 1191 